\title{
Abundances and Physical Conditions in the Interstellar Gas toward HD 192639. ${ }^{1}$
}

\author{
P. Sonnentrucker ${ }^{2}$, S. D. Friedman ${ }^{2}$, D. E. Welty ${ }^{3}$, D. G. York ${ }^{3}$ and T. P. Snow ${ }^{4}$
}

\begin{abstract}
We present a study of the abundances and physical conditions in the interstellar gas toward the heavily reddened star HD $192639\left[E_{(B-V)}=0.64\right]$, based on analysis of FUSE and HST/STIS spectra covering the range from 912 to $1361 \AA$. This work constitutes a survey of the analyses that can be performed to study the interstellar gas when combining data from different instruments. Low-velocity ( -18 to $-8 \mathrm{~km} \mathrm{~s}^{-1}$ ) components are seen primarily for various neutral and singly ionized species such as C I, O I, S I, Mg II, Cl I, Cl II, Mn II, Fe II and $\mathrm{Cu}$ II. Numerous lines of $\mathrm{H}_{2}$ are present in the FUSE spectra, with a kinetic temperature for the lowest rotational levels $T_{01}=(90 \pm 10) \mathrm{K}$. Analysis of the $\mathrm{C}$ I fine-structure excitation implies an average local density of hydrogen $n_{\mathrm{H}}=(16 \pm 3) \mathrm{cm}^{-3}$. The average electron density, derived from five neutral/first ion pairs under the assumption of photoionization equilibrium, is $n_{e}=(0.11 \pm$ $0.02) \mathrm{cm}^{-3}$. The relatively complex component structure seen in high-resolution spectra of K I and Na I, the relatively low average density, and the measured depletions all suggest that the line of sight contains a number of diffuse clouds, rather than a single dense, translucent cloud. Comparisons of the fractions of $\mathrm{Cl}$ in $\mathrm{Cl} \mathrm{I}$ and of hydrogen in molecular form suggest a higher molecular fraction, in the region(s) where $\mathrm{H}_{2}$ is present, than that derived considering the average line of sight. In general, such comparisons may allow the identification and characterization of translucent portions of such complex lines of sight. The combined
\end{abstract}

\footnotetext{
1 "Based on observations made with the NASA/ESA Hubble Space Telescope, obtained from the Data Archive at the Space Telescope Science Institute, which is operated by the Association of Universities for Research in Astronomy, Inc., under NASA contract NAS 5-26555. These observations are associated with proposal \#8241."

${ }^{2}$ Department of Physics and Astronomy, Johns Hopkins University, 3400 North Charles Street, Baltimore, MD 21218

${ }^{3}$ Department of Astronomy and Astrophysics, University of Chicago, 5640 South Ellis Avenue, Chicago, IL 60637 .

${ }^{4}$ Center for Astrophysics and Space Astronomy, University of Colorado, Campus Box 389, Boulder, CO 80309
} 
data also show high-velocity components near $-80 \mathrm{~km} \mathrm{~s}^{-1}$ for various species which appear to be predominantly ionized, and may be due to a radiative shock. A brief overview of the conditions in this gas will be given.

Subject headings: ISM: abundances, atoms-ISM: clouds, molecules - stars: individual (HD 192639)

\section{Introduction}

The Far Ultraviolet Spectroscopic Explorer (FUSE) allows us to study $\mathrm{H}_{2}$ directly in the ground state absorption lines. Its wavelength coverage (912-1187 $\AA$ ) includes numerous electronic transitions from the strong $\mathrm{H}_{2}$ Werner and Lyman bands as well as from a wealth of heavy elements crucial for diagnosing the temperature, abundance, depletion and density of the interstellar gas. Furthermore, the FUSE sensitivity permits the observation of fainter stars with higher extinctions than previously possible with far-UV instruments (Moos et al. 2000). Hence, FUSE offers a unique opportunity to study the properties of clouds with visual extinction $A_{V}$ greater than 1 magnitude (mag), defined as translucent clouds. Little is known about the UV absorption-line properties of such clouds since their observation was technically difficult until the FUSE era. What are the dominant constituents of translucent clouds apart from molecular hydrogen? How severe is the depletion in such clouds? At what temperature and density is the gas in such clouds? What characterizes the transition from diffuse to translucent clouds?

The FUSE translucent cloud program was designed to answer these questions. It involves more than 30 stars chosen for their values of $A_{V}$ ranging from 1 to 5 mag in an effort to distinguish translucent clouds from ensembles of diffuse clouds, to determine abundances, depletions, and physical conditions in those clouds, and to search for clouds presenting properties intermediate between diffuse and translucent (see HD 110432 in Rachford et al. 2001a). HD 192639 is among the stars selected in the FUSE translucent cloud program for its extinction $\left(A_{V} \sim 2\right)$, typical of that class of clouds. This star was also observed with HST in "The Snapshot Survey of Interstellar Absorption Lines" conducted by J. Lauroesch (P8241).

HD 192639 is an O7.5 $\mathrm{Ib}(\mathrm{f})$ (Walborn 1972) star bearing a reddening $\mathrm{E}_{(B-V)}=0.64$. This star is a member of the Cyg OB1 Association, one of the most luminous OB associations in the solar neighborhood. The association is centered at $\left(l^{I I}, b^{I I}\right)=\left(76^{\circ}, 1^{\circ}\right)$ at an estimated distance of $1.8 \mathrm{kpc}$ (St-Louis \& Smith 1991). Previous studies of the Cygnus region have 
shown it to be dynamically active, with shocked gas and related H II regions being detected at high and intermediate velocity toward Cyg OB1 and OB3 (Phillips et al. 1984; St-Louis \& Smith 1991; Lozinskaya et al. 1998a). Equivalent width estimates were obtained from IUE data for a few heavy elements (Phillips et al. 1984; St-Louis \& Smith 1991), and molecular hydrogen $\left(\mathrm{H}_{2}\right)$ column densities were indirectly derived from infrared measurements which are sensitive only to the highest density regions.

We report here the far ultraviolet (FUV) study of the potentially translucent cloud line of sight toward HD 192639. We present a survey of the analyses that can be performed when combining data from different instruments. Such combinations allow us to detect a wide range of species in various ionization states and to perform detailed studies of the local conditions in the ISM gas. This study focuses on the detailed analysis of the cold interstellar gas toward HD 192639 but will also briefly give an overview of the other gas components detected in the combined datasets along this line of sight. This work comprises the analysis of the FUV absorption lines included in the combined FUSE and archival HST/STIS datasets covering the wavelength range 912-1361 $\AA$. The resolutions of $18 \mathrm{~km} \mathrm{~s}^{-1}$ (FUSE low-resolution aperture) and $2.75 \mathrm{~km} \mathrm{~s}^{-1}$ (STIS E140H), supported by high-resolution optical $\mathrm{K} \mathrm{I}$ and $\mathrm{Na}$ I data $\left(\sim 1.5-1.6 \mathrm{~km} \mathrm{~s}^{-1}\right)$, allowed us to derive accurate column densities for many important gas-phase species, among which are C I, C I*, C I**, N II, O I, O I*, O I**, Mg II, Si II, S I, Cl II, Mn II, Fe II and $\mathrm{H}_{2}$. The data reduction is described in $\S 2$. A study of the velocity structure toward HD 192639 is found in $\S 3$. The physical properties of the low-velocity gas in terms of pressure, temperature, density and depletion are derived and discussed in $\S 4$. A brief study of the high-velocity gas detected with the combined data toward HD 192639 in the C II, C II*, N II, N II*, Si II, Si II* and S II lines is presented in $\S$ 5 and the main results of this work are summarized in $\S 6$.

\section{Data processing and analysis}

FUSE observed HD 192639 (Program P1162401) in June 2000, in time tag mode (Sahnow et al. 2000) for a total of 4834s with the low resolution aperture (LWRS), covering the wavelength range 912-1187 $\AA$. The data resolution is about $0.062 \AA$ (FWHM, corresponding to $\sim 18 \mathrm{~km} \mathrm{~s}^{-1}$ and $\sim 9$ pixels). No detector burst activity was found in either of the two observations (Sahnow et al. 2000). The data were processed with version 1.8.7 of the CALFUSE pipeline which included geometric distortion correction, Doppler correction to set the heliocentric rest-frame, dead-time correction and wavelength calibration (Kruk \& Murphy 2001; Sahnow et al. 2000). The exposures were co-added after cross-correlating and shifting 
the spectra with respect to the brighter exposure, for each channel. The shifts are 8 pixels maximum. The LiF1B spectrum was excluded from the data analysis due to the presence of a well known detector artifact causing an artificial flux deficiency in this segment (Kruk \& Murphy 2001). Figure 1 presents a sample of the molecular hydrogen lines used to derive the column densities in the rotational states $J=0$ to $J=5$. One of the windows used to fit each transition ( panels show selected lines for each rotational transition in the heliocentric rest-frame. The spectra used for the data analysis were binned by 4 pixels (less than one resolution element) in order to increase the signal-to-noise $(\mathrm{S} / \mathrm{N})$ ratio without degrading the resolution. The co-added spectra have $\mathrm{S} / \mathrm{N}$ of about 10 per resolution element in the LiF1A spectrum and up to 15 per resolution element in the LiF2A spectrum. Weak lines such as those from Cl II, $\mathrm{C} \mathrm{I}^{* *}$ or Fe II were assumed to be positively detected (and not the result of fixed pattern noise contamination) when the lines were present in more than one channel.

The STIS data for HD 192639 were obtained for "The Snapshot Survey of Interstellar Absorption Lines" (P8241) conducted by J. Lauroesch using the FUV MAMA detector and the $0 . " 2 \times 0 . " 2$ aperture. The E140H echelle grating provides a resolution of $2.75 \mathrm{~km} \mathrm{~s}^{-1}$ and spans the wavelength range between 1150-1361 $\AA$, therefore complementing the FUSE bandpass. The 49 extracted orders were corrected for scattered light contamination following the procedure described in Howk \& Sembach (2000).

High-resolution (FWHM $\sim 1.5-1.6 \mathrm{~km} \mathrm{~s}^{-1}$ ) spectra of $\mathrm{K} \mathrm{I}, \mathrm{Na}$ I, Ca I, CH, and $\mathrm{CH}^{+}$ were obtained with the Kitt Peak coudé feed telescope in 1998. A detailed discussion of the reduction and analyses of these spectra - and of similar spectra for other stars in the FUSE translucent cloud survey (Rachford et al. 2001b) — will be given by Welty et al. (2002b in prep.). The spectra of K I and Na I are shown in Fig. 2 along with lines detected with STIS and FUSE.

HD 192639 was observed with the echelle spectrographs on board the IUE satellite at a resolution of 0.1-0.2 $\AA$ for a total of $226 \mathrm{~min}$. Figure 5 shows the C IV and Si IV lines detected in the short wavelength image (SWP 9493) which resulted from an integration of 136 min (Phillips et al. 1984). The data shown here were retrieved from the MAST archival database and used without any additional processing.

The absorption lines used in the following analysis are shown in Figs. 1 through 6. Each species of interest is indicated by a series of 5 tick marks positioned at the velocities of each 
one of the 5 components detected in the low-velocity gas (LVCs, see $\S 3$ ). The corresponding wavelength of each species is noted at the top right of each panel. No shifts were necessary to align the STIS data with the ground based data. The FUSE data were shifted with respect to the STIS data by $-16 \mathrm{~km} \mathrm{~s}^{-1}$ (LiF1A), $-10 \mathrm{~km} \mathrm{~s}^{-1}$ (LiF2A) and $+31 \mathrm{~km} \mathrm{~s}^{-1}$ (SiC2B; $\mathrm{SiC} 1 \mathrm{~A})$. A shift of $-10 \mathrm{~km} \mathrm{~s}^{-1}$ was applied to the $I U E$ data to align the S II and Si II lines with the corresponding STIS lines. If high-velocity components (HVCs) are detected (see $\S$ $3)$, an additional tick mark is drawn at their average position $\left(-80 \mathrm{~km} \mathrm{~s}^{-1}\right)$. All velocities are heliocentric. When other absorption lines are present in the velocity range considered, those elements are noted below the spectrum. The source of the data is indicated at the bottom right of each panel as follows: (I) for IUE data, (F) for FUSE data, (O) for optical data and $(\mathrm{S})$ for STIS data. The continua for the lines were normalized to unity, in Figs. 2 to 6 , by applying low-order polynomials to the spectral regions adjacent to each absorption line.

\section{Line-of-sight velocity structure}

The detailed interstellar component velocity structure was inferred from the highresolution K I and Na I spectra. Fits to the K I line profile indicate (at least) five components, at velocities from -18 to $-8 \mathrm{~km} \mathrm{~s}^{-1}$, which comprise the three-component groups discernible in the lower resolution STIS spectra of species such as C I, O I, S I, Mg II, or Ni II (Figs. 2, 3 and 4). For T $100 \mathrm{~K}$ (see below), the K I $b$-values $\left(0.8-1.2 \mathrm{~km} \mathrm{~s}^{-1}\right)$ suggest that the line broadening is dominated by turbulence for each of the components. These three-component groups seen in STIS contain predominantly neutral (H I) gas, most of the $\mathrm{H} \mathrm{I}$ and $\mathrm{H}_{2}$ along this line of sight, and will be referred to as the Low-Velocity Components (LVCs).

Some of the stronger UV lines (e.g., of C II, Si II, and Si III) reveal absorption from high-velocity gas near $-80 \mathrm{~km} \mathrm{~s}^{-1}$; at least two components can be discerned in the profiles of Si II* and S II. Spectra from IUE show absorption from yet higher ions (C IV, Si IV, Al III, S III) at those velocities, indicating that the High-Velocity Components (HVCs) are predominantly ionized (Phillips et al. 1984). We note, however, that high-velocity absorption is also seen for N I, and Na I - so that some neutral gas is present as well.

While we focus in the next sections on the abundances and physical conditions in the LVCs and briefly discuss the HVCs, we note that several relatively weak components, at velocities from $-27 \mathrm{~km} \mathrm{~s}^{-1}$ to $+12 \mathrm{~km} \mathrm{~s}^{-1}$, are seen in $\mathrm{Na}$ I (and in the UV lines of various other species). Additional intermediate-velocity components, extending to about $-60 \mathrm{~km}$ $\mathrm{s}^{-1}$, are present in the stronger UV lines of C II, N I, O I, and Si II. These components likely 
contain mixtures of neutral and ionized gas; the absorption at $+10 \mathrm{~km} \mathrm{~s}^{-1}$ may largely arise in an H II region (Phillips et al. 1984; St-Louis \& Smith 1991).

\section{The Low-Velocity Components (LVCs)}

The equivalent widths of the heavy elements measured over the velocity range -30 to $0 \mathrm{~km} \mathrm{~s}^{-1}$ are summarized in Table 1 . Wavelengths and $f$-values were taken from (Morton 2001 in prep.) unless noted otherwise in Table 1. The column densities of the species present in both FUSE and STIS datasets were derived using up to four different methods depending on the type and number of transitions available; 1) a curve of growth (COG) analysis, 2) an apparent optical depth (AOD) analysis for optically thin or slightly saturated lines, 3) Equivalent Width (EW) analysis for optically thin lines and 4) a profile-fitting (FIT) analysis based on the "Owens" code described by Lemoine et al. (2001). When multiple methods were used and showed consistent results, we calculated the weighted mean of the column densities, weighting each column density by its error for a given species. The results and associated $1 \sigma$ errors are reported in Table 3. The methods used to derived the column densities are indicated in column (3) of Table 3.

\subsection{Component analysis of the LV gas}

Lines from a number of neutral and ionized species present in the STIS spectra, C I and S I, exhibit profiles similar to that of K I. Because the profile analysis of the high-resolution $\mathrm{K}$ I lines shows that the gas motion in the LVCs is dominated by turbulence at the typical temperature of the cold interstellar medium $(100 \mathrm{~K})$, we used the K I component structure to fit the STIS C I, C I*, C I**, O I, Mg II, S I, Cl I, Mn II, Fe II, Ni II, Cu II and Ge II profiles. The number of components and $b$-values were held fixed, while the component column densities and the continuum were allowed to vary. Because the five components detected in the high-resolution K I spectra are both narrow $\left(b \sim 0.8-1.2 \mathrm{~km} \mathrm{~s}^{-1}\right)$ and separated from

each other by only $2-3 \mathrm{~km} \mathrm{~s}^{-1}$, they are only partially resolved in the STIS spectra. In such cases, even the relatively small uncertainties in velocity zero point in the STIS spectra can lead to large uncertainties in the column densities of some individual components. While the total line-of-sight LV column densities are fairly well determined, some of the individual LV component column densities listed in Table 2 thus are known only to within a factor of 2. Given those uncertainties, it is difficult to distinguish differences in the abundances, depletions, pressures, and densities among the individual LV components. The gas in all five components seems to exhibit similar conditions suggesting that each component contributes 
similarly to the total pressure, density, abundances and depletions over the entire LVCs. In light of the generally good correlation between $N(\mathrm{CH})$ and $N\left(\mathrm{H}_{2}\right)$ in interstellar clouds (Danks, Federman, \& Lambert 1984), the roughly constant ratio $N(\mathrm{~K} \mathrm{I}) / N(\mathrm{CH})$ found for the five LV components suggests that each component also contributes similarly to $\mathrm{H}_{2}(J=$ 0 to 2 ) and (perhaps) to CO.

The low resolution (FWHM $\sim 18 \mathrm{~km} \mathrm{~s}^{-1}$ ) and small-scale wavelength uncertainties in the FUSE spectra preclude detailed component analyses for such species as $\mathrm{H}_{2}, \mathrm{Cl}$ II, Fe II, and CO. The generally similar properties seen for the individual LV components in the higher resolution optical and STIS spectra, however, suggest that, in this particular case, reasonably accurate total line-of-sight column densities may be obtained from the FUSE spectra by using either single-component fits or else multi-component fits in which the relative LV component column densities (as well as the $b$-values and relative velocities) are all held fixed. This approximation is well-supported by the fact that no inconsistencies were found between the one-component COG, 5-component FIT and AOD results derived for the LV gas species detected in the STIS data, as illustrated by the analysis of neutral carbon (see Fig. 7 and $\S 4.3$ ). [That was not the case for HD 73882 (Snow et al. 2000), where the individual components differed more — both in overall strength and in ratios such as $N(\mathrm{~K} \mathrm{I}) / N(\mathrm{CH})$.] In light of these results, the following study of the physical properties of the cold interstellar gas toward HD 192639 will be based on the total LV column densities.

\subsection{Molecular lines}

The FUSE wavelength range contains numerous $\mathrm{H}_{2}$ lines from the Werner and Lyman bands. Because of the large column densities, the $J=0$ to 2 rotational transitions are severely blended with each other, so that their column densities can only be derived using profile fitting techniques. On the other hand, the $J=3$ to 5 rotational transitions can be analyzed either via profile fitting or via curve of growth techniques. Rachford et al. (2001b) performed a multi-component curve of growth analysis of the $\mathrm{H}_{2}$ lines as part of the $\mathrm{H}_{2}$ translucent cloud survey. We performed a profile fitting analysis for all $\mathrm{H}_{2}$ lines, in part to compare results from the two independent methods toward HD 192639. The $\mathrm{H}_{2}$ column densities summarized in Table 3 arose from a single-component Voigt profile fitting of the lines from the $J=0$ to $J=5$ rotational levels in the FUSE bandpass. All lines were simultaneously fitted in the 1043-1055, 1073-1083 and 1088-1098 $\AA$ windows present in the LiF1A, LiF2A and SiC1A segments (see Fig. 1, segment LiF1A). As for the atomic species, consistency was found between the COG results from Rachford et al. (2001b) and our profile 
fits. These analyses showed that the $J=0$ to 2 lines are damped and that the $J=5$ lines are optically thin whereas the $J=3$ and 4 lines show various degrees of saturation. The best fit (minimized $\left.\chi^{2}\right)$ led to $b=(7.2 \pm 0.3) \mathrm{km} \mathrm{s}^{-1}$ (constrained by the $J=3$ and 4 lines) using a line spread function of 9 pixels for the FUSE data. For a check of consistency, we also performed a 5-component fit of the $J=0$ to 2 levels using the $\mathrm{K}$ I structure (see $\S$ 4.1) and, again, found no discrepancy between the total column densities derived from the 5-component fit and those obtained with the single-component profile fit. The excitation diagram $\left(\mathrm{N}(J) / \mathrm{g}_{J}\right.$ versus $\left.\mathrm{E}_{J}\right)$ led to a kinetic temperature derived from the $J=0$ and $J=$ 1 rotational levels of $\mathrm{T}_{01}=(90 \pm 10) \mathrm{K}$. The diagram also showed that the $J=2$ level also appears to be in thermal equilibrium at $\mathrm{T}_{01}$, suggesting that the population of that level is still dominated by collisions with thermal atoms. This analysis resulted in a total $\mathrm{H}_{2}$ column density of $(20.76 \pm 0.09)$ dex and a total molecular fraction of $f \equiv 2 \mathrm{~N}\left(\mathrm{H}_{2}\right) /[\mathrm{N}(\mathrm{H} \mathrm{I})$ $\left.+2 \mathrm{~N}\left(\mathrm{H}_{2}\right)\right]=(0.36 \pm 0.10)$ in the LV gas. We used the H I column density measurement of $(21.32 \pm 0.12)$ dex from Diplas \& Savage (1994). The higher $J$ levels are not in thermal equilibrium at $\mathrm{T}_{01}$ indicating that those transitions are, most probably, predominantly populated by UV pumping (Black \& Dalgarno 1973). One might expect a broader velocity distribution for those higher levels as indicated by the larger $b$-value found for $\mathrm{H}_{2}\left(7 \mathrm{~km} \mathrm{~s}^{-1}\right.$ from $J=3$ and 4 vs $4 \mathrm{~km} \mathrm{~s}^{-1}$ for C I; see $\S 4.3$ ) if the excited $\mathrm{H}_{2}$ arises from less shielded parts of the clouds. Comparable $b$-values were found and discussed by Jenkins \& Peimbert (1997) in the case of $\zeta$ Ori.

Carbon monoxide was detected in the FUSE and STIS datasets at 1076, 1087 and 1344 $\AA$. The detailed discussion of the molecular content toward HD 192639 (including the study of the higher $J$ levels of molecular hydrogen) is addressed in a paper in preparation which compares HD 192639 to another sightline and discusses the variability of the $\mathrm{CO} / \mathrm{H}_{2}$ ratio (Sonnentrucker et al. 2002 in preparation). We will therefore only mention the main results of the CO analysis toward HD 192639 here. The detailed profile analysis of the 1076 and $1344 \AA$ bands showed that the latter is optically thin and the former is slightly saturated. The $1087 \AA$ band was not used due to its blend with the $1088 \AA \mathrm{Cl}$ I line (see $\S 4.6)$. An estimate of its equivalent width, is, however, given in Table 1 . The optically thin $1344 \AA$ band is very weak but shows the component groups seen in K I, C I or S I characteristic of the cold neutral gas. The analysis of the rotational transitions indicates that the band is dominated by the $\mathrm{R}(0)$ transition. A small contribution of the $J=1$ transitions is however suggested by the 5-component fit based on the K I structure (see $\S 4.1$ and Table 1). Again, the low resolution of the FUSE data and the extreme weakness of the $1344 \AA$ band did not allow the study of the $\mathrm{CO}$ distribution on a component-by-component basis. Table 3 , hence, contains the total line-of-sight $\mathrm{CO}$ column density derived from the $J=0$ and 1 transitions 
using the 1076 and $1344 \AA$ bands. We adopted the wavelengths and $f$-values from Morton \& Noreau (1994) for the $1344 \AA$ band and Federman et al. (2001) for the $1076 \AA$ band.

\subsection{Local hydrogen density: $n_{\mathrm{H}}$}

Absorption from the ground and excited fine-structure states of C I is only detected in the LVCs. The large sample of lines (24 in all) available in the combined FUSE and STIS data allowed us to use three of the analysis methods mentioned above and thus to derive accurate column densities for $\mathrm{C} \mathrm{I}, \mathrm{C} \mathrm{I}^{*}$, and $\mathrm{C} \mathrm{I}^{* *}$ as follows:

- Because the two trace species K I and C I should largely coexist, we used the component structure derived from the high-resolution optical spectra of K I to fit the lines of $\mathrm{C}$ I and excited states seen in the STIS spectra. As noted above, for $T \sim 100 \mathrm{~K}$ the component $b$-values (for both species) are dominated by turbulence. We, hence, adopted the $b$-values of $\mathrm{K}$ I as fixed parameters. The free parameters in the C I, C I* and $\mathrm{C} \mathrm{I}^{* *}$ fits were the 5 individual component column densities and the continuum. The component-by-component results are summarized in Table 2. This fitting procedure was also used on the FUSE C I lines. The total column density derived with the FUSE data was consistent with the STIS results within $2 \sigma$. The carbon excited states in the FUSE data could not be fitted owing to the lower resolution and lower $\mathrm{S} / \mathrm{N}$ of the data as well as strong blends with other lines.

- The equivalent widths of the C I, C I* and C I** lines were measured (Table 1) and fitted to separate curves of growth (Fig. 7). The column densities, $b$-values and respective $1 \sigma$ errors for each species are reported in each panel. The total column densities derived from the curves of growth are consistent with those derived from the detailed profile fits within $1 \sigma$. For the lines we measured, single-component curves of growth with effective $b$-values of $4 \mathrm{~km} \mathrm{~s}^{-1}$ provide reasonable representations of the more complex reality; they are consistent with curves generated using the $\mathrm{K} \mathrm{I}$ and $\mathrm{Na} \mathrm{I}$ component structures. We note that use of the $f$-values suggested by Jenkins \& Tripp (2001) would lead to curves of growth with effective $b \gtrsim 5 \mathrm{~km} \mathrm{~s}^{-1}$ and significantly lower column densities.

- We also integrated the apparent optical depth of the weak C I (1270.14 $\AA)$, C I* $(1276.75 \AA)$ and $\mathrm{C} \mathrm{I}^{* *}(1261.43 \AA)$ lines over the velocity range -30 to $0 \mathrm{~km} \mathrm{~s}^{-1}$ assuming unresolved saturated structures were not present. This last set of results was also in agreement with the fit and COG results within $1 \sigma$. 
As mentioned in $\S 4.1$, the 3 methods gave consistent results within errors which indicates that a single-component analysis provides a fair approximation of the more complicated highresolution situation, in the particular case of HD 192639. From our measurements, the total fractional abundances of carbon in the excited states are: $\mathrm{f}_{1} \equiv N\left(\mathrm{C} \mathrm{I}^{*}\right) / N\left(\mathrm{C}_{\text {total }}\right)=0.134$ and $\mathrm{f}_{2} \equiv N\left(\mathrm{C} \mathrm{I}^{* *}\right) / N\left(\mathrm{C}_{\text {total }}\right)=0.020$, leading to a pressure estimate $\log (P / k)=\log \left(n_{\mathrm{H}} T\right)$ between 3.1 and 3.3 for the LV gas (Jenkins \& Shaya 1979), if the gas is bathed in the average interstellar radiation field. Assuming a kinetic temperature for carbon of $100 \mathrm{~K}$, in agreement with that of $\mathrm{H}_{2}\left(\mathrm{~T}_{01}\right)$, the number density of neutral hydrogen atoms (averaged over the 5 components) $n_{\mathrm{H}}$ is estimated to range from 12 to $20 \mathrm{~cm}^{-3}$ in the LVCs. This range implies that the $\mathrm{H}$ I-containing gas has a total thickness between $\sim 34$ and $57 \mathrm{pc}$.

The $1355 \AA$ O I line and the excited fine-structure states O I* (1304 $\AA)$ and O I** (1306 $\AA$ ) were also detected in the LV gas (Fig. 3). We derived the O I column density using the methods described earlier for neutral carbon. The $\mathrm{O} \mathrm{I}^{*}$ and $\mathrm{O} \mathrm{I}^{* *}$ lines are assumed optically thin allowing derivation of their respective column densities from the equivalent width measurements. A comparison of the profiles (Fig. 6), however, suggests that the column density distribution in $\mathrm{O} \mathrm{I}^{*}$ and $\mathrm{O} \mathrm{I}^{* *}$ is different from that in $\mathrm{O}$ I or C I. In order to quantify these profile variations, we performed tests by fitting the $\mathrm{O} \mathrm{I}^{*}$ and $\mathrm{O} \mathrm{I}^{* *}$ profiles using $\mathrm{O}$ I and $\mathrm{C}$ I as the baseline for the initial 5 -component structures. The $b$-values were fixed to those of $\mathrm{K} \mathrm{I}$ for each component (see $\S 4.1$ ). The total column densities are consistent with the column densities derived from the equivalent width analysis (see Tables 2 and 3). We, therefore, reported the weighted mean of the column densities for O I, O I* and $\mathrm{O} \mathrm{I}^{* *}$ in Table 3. We adopted the latter total column densities, and used the Keenan \& Berrington (1988) calculations of the fine-structure population ratios, to estimate the neutral hydrogen volume density in the LVCs where O I is detected. We find population ratios of $\log \left[\mathrm{f}_{1} \equiv N\left(\mathrm{O} \mathrm{I}^{*}\right) / N\left(\mathrm{O}_{\text {total }}\right)\right]=-5.25$ and $\log \left[\mathrm{f}_{2} \equiv N\left(\mathrm{O} \mathrm{I}^{* *}\right) / N\left(\mathrm{O}_{\text {total }}\right)\right]=-5.43$. The best match with Keenan \& Berrington (1988) calculations occurs for $\mathrm{T}=100 \mathrm{~K}$ and $11 \leq n_{\mathrm{H}} \leq 26 \mathrm{~cm}^{-3}$, which is consistent with the volume density derived from the C I analysis. The C I and O I volume densities are of the order of those found in diffuse clouds, indicating that the LVCs are most probably dominated by diffuse cloud gas. The componentby-component analysis (Table 2) suggests that potentially slightly denser material is concentrated in component 3 in the LVCs. However, the weakness of the $\mathrm{O} \mathrm{I}^{* *}$ line, the poor $\mathrm{S} / \mathrm{N}$ in the line and the uncertainties linked to the individual column density determinations (see $\S 4.1$ ) prevent us from discerning whether the $\mathrm{O} \mathrm{I}^{* *}$ line profile resembles that of O I* or is different. New high-resolution and high $\mathrm{S} / \mathrm{N}$ observations of those atomic lines are warranted to confirm the profiles presented in this study as well as the potentially denser neutral hydrogen clumping. 


\subsection{Depletion}

Except for C I, Na I, Mg I, S I and K I, the species detected in the neutral gas are in their dominant charge state, allowing us to derive their depletions. We used the solar abundances from Anders \& Grevesse (1989) and Grevesse \& Noels (1993) to estimate the depletions of $\mathrm{Mg}, \mathrm{Cl}, \mathrm{Mn}, \mathrm{Fe}, \mathrm{Ni}, \mathrm{Cu}$ and $\mathrm{Ge}$. The solar abundance for $\mathrm{O}$ was taken from Holweger

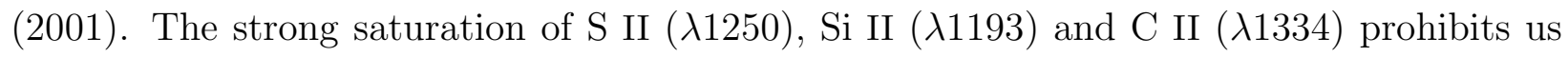
from deriving any information on the depletion of these species. We will, however, assume abundances to be solar for sulfur and adopt the fairly constant interstellar carbon depletion of -0.4 dex from solar (Sofia et al. 1997) in the following discussion on electron densities. Table 2 and Fig. 4 show that all refractory elements seen in STIS exhibit very similar profiles suggesting that abundances and depletions are very similar in each component. Hence, as in the case of the trace neutrals, the total LV gas abundances and depletions can and will be considered. Table 4 summarizes the abundances relative to hydrogen and the depletions for the LV gas (over the 5 components). Figure 8 compares the estimated depletions with the average depletions for the typical "cold" and "warm" clouds adopted by Welty et al. (1999; see references therein). If the HD 192639 line of sight traversed a translucent cloud, one would expect the depletions to be of the order of, or more severe, than those in the cold diffuse clouds (Snow 1975). However, the LV gas does not show enhanced depletions of the species forming dust grains (Mg II, Mn II, Fe II, Ni II or Cu II) but rather shows depletions intermediate between cold and warm diffuse clouds. Therefore, even though the line of sight toward HD 192639 has been considered as a candidate for containing a translucent cloud on the basis of its reddening, this depletion study supports the diffuse-cloud dominated nature of the LV gas, already suggested by the complexity of the velocity structure shown in the trace neutral species and the volume density derived from the carbon analysis. This line of sight should therefore be called "translucent line of sight" rather than "translucent cloud" (Rachford et al. 2001b).

\subsection{Electron Density and Fractional Ionization}

We used neutral/first ion pairs for five elements (C, Na, Mg, S and K) and the assumption of photoionization equilibrium to estimate the electron density in the LVCs (Table 5). Except for $\mathrm{Mg}$, we do not have measured column densities for the dominant first ions, and so we estimated values based on assumed depletions: 0.0 dex for S (Spitzer \& Fitzpatrick 1993), -0.4 dex for C (Sofia et al. 1997), -0.6 dex for K, and -0.7 dex for Na. The values for $\mathrm{K}$ and $\mathrm{Na}$ were estimated based on considerations of photoionization equilibrium (Welty \& Hobbs 2001), and may be too severe if other processes (e.g., charge exchange with large 
molecules; Weingartner \& Draine 2001) affect the ionization balance of the heavy elements. The photoionization rates were calculated using the average IS radiation fields of Draine (1978) and Witt \& Johnson (1973); the recombination rates were calculated for $T=100$ K (Péquignot \& Aldrovandi 1986). The electron densities inferred from C, Na, Mg, and K are fairly similar $(0.11 \pm 0.02) \mathrm{cm}^{-3}$; the much lower value from $\mathrm{S}$ has been noted in other sightlines (Welty et al. 1999; Welty et al. 2002a).

On the basis of the carbon analysis and the previous depletion discussion, we adopted a mean electron density of $(0.11 \pm 0.02) \mathrm{cm}^{-3}$ and mean neutral hydrogen density of $(16$ $\pm 3) \mathrm{cm}^{-3}$ indicating an average ionization fraction $n_{e} / n_{\mathrm{H}} \sim 0.007$, which is higher than that expected from the carbon ionization only $\left(1.4 \times 10^{-4}\right)$. Such high fractional ionizations have been seen in other sightlines (e.g., 23 Ori; Welty et al. 1999; Welty et al. 2002a), and may be due (at least in part) to overestimation of $n_{e}$ if processes such as charge exchange with large molecules are significant (Lepp et al. 1988; Weingartner \& Draine 2001). On the other hand, the presence of N II and maybe N II* in the LVCs also suggests there is some ionized gas amid the neutral gas which could partly explain the high ionization fraction toward HD 192639.

\subsection{Chlorine chemistry}

In regions where $\mathrm{H}_{2}$ is optically thick, $\mathrm{Cl}$ II reacts rapidly with $\mathrm{H}_{2}$ to form $\mathrm{HCl}^{+}$, which in turn (via several routes) leads to $\mathrm{Cl} \mathrm{I}$ and $\mathrm{H} \mathrm{I}$. Because the conversion of $\mathrm{Cl} \mathrm{II}$ to $\mathrm{Cl} \mathrm{I}$ is faster (rate constant $\left.\mathrm{k}=7 \times 10^{-10} \mathrm{~cm}^{3} \mathrm{~s}^{-1}\right)$ than the photoionization of $\mathrm{Cl} \mathrm{I}\left(\Gamma=2 \times 10^{-12}\right.$ $\mathrm{s}^{-1}$ ), $\mathrm{Cl}$ is primarily neutral in regions where $\mathrm{H}_{2}$ is abundant, but otherwise is primarily ionized (Jura 1974; Jura \& York 1978). This behavior is unique to Cl. Jura \& York proposed a simple cloud model consisting of two zones: 1) a region where $\mathrm{H}_{2}$ is optically thick, with $\mathrm{H}_{2}$, Cl I, and some H I, and 2) a region where $\mathrm{H}_{2}$ is optically thin, dominated by $\mathrm{H}$ I and $\mathrm{Cl}$ II. In principle, each cloud along a line of sight could have these two zones. Equation A7 in Jura \& York then implies that $N_{1}(\mathrm{H} \mathrm{I})=N_{\mathrm{H}}\left[f(\mathrm{Cl} \mathrm{I})-f\left(\mathrm{H}_{2}\right)\right]$, where $N_{1}(\mathrm{H} \mathrm{I})$ is the column density of $\mathrm{H}$ I in the optically thick region, $N_{\mathrm{H}}$ is the total hydrogen (atomic plus molecular) column density for the line of sight and $f(\mathrm{Cl} \mathrm{I})$ and $f\left(\mathrm{H}_{2}\right)$ are the fractions of $\mathrm{Cl}$ in neutral form and of hydrogen in molecular form, respectively, averaged over the line of sight.

Chlorine lines were detected in the LVCs at 1004, 1088, 1097 (Cl I), and $1071 \AA$ (Cl II) 
with FUSE and at $1347 \AA$ (Cl I) with STIS; several other weak Cl I lines may be present in the FUSE spectrum. The Cl II column density was estimated using the equivalent width and with the AOD method, assuming the line is optically thin. Both methods gave results consistent with $N(\mathrm{Cl} \mathrm{II}) \sim 1.7 \pm 0.4 \times 10^{14} \mathrm{~cm}^{-2}$. All three of the $\mathrm{Cl}$ I lines are somewhat saturated; the line at $1088.059 \AA$ is slightly blended with a CO line at $1087.868 \AA$ (see Fig. 2). Unfortunately, only the $\mathrm{Cl}$ I lines at 1088, 1097, and $1347 \AA$ have fairly reliable, experimentally determined $f$-values (Schectman et al. 1993). Application of the AOD method to the $\lambda 1347$ line implies $N(\mathrm{Cl} \mathrm{I})>1.1 \times 10^{14} \mathrm{~cm}^{-2}$. Fits to the FUSE lines, using the component structure derived from the higher resolution K I and $\mathrm{Na}$ I data, with various constraints on the relative column densities of the five strongest components, suggest that $N(\mathrm{Cl}$ I $) \sim 1.1-1.5 \times 10^{14} \mathrm{~cm}^{-2}$. Fits to the $\lambda 1347$ line, using the same component structure and constraints on the relative column densities of the five strongest components, suggest that $N(\mathrm{Cl} \mathrm{I}) \sim 2-3 \times 10^{14} \mathrm{~cm}^{-2}$. Those combinations of component structure and total column density yield $\mathrm{W}(1088) \sim 50 \mathrm{~m} \AA$ (vs. the observed $50 \pm 15 \mathrm{~m} \AA$ ), but appear to somewhat overestimate the strength of the weaker FUSE $\lambda 1097$ line. In the light of the AOD and FIT results, we will adopt $N(\mathrm{Cl} \mathrm{I}) \sim 2.0_{-0.5}^{+1.0} \times 10^{14} \mathrm{~cm}^{-2}$.

The adopted column densities imply $f(\mathrm{Cl} \mathrm{I})=0.54$ - similar to the values found in a survey of less heavily reddened sightlines, for comparable $f\left(\mathrm{H}_{2}\right)$ (Jenkins et al. 1986). With $f\left(\mathrm{H}_{2}\right)=0.36$, we find $N_{1}(\mathrm{H} \mathrm{I})=5.8 \times 10^{20} \mathrm{~cm}^{-2}$ - so that about $28 \%$ of the $\mathrm{H}$ I is in zone(s) 1 , where $\mathrm{H}_{2}$ is abundant and optically thick. The $f\left(\mathrm{H}_{2}\right)$ in zone(s) 1 would then be about 0.66 - i.e., higher than the average value 0.36 for the whole line of sight, but somewhat lower than the values expected for translucent clouds. If $N\left(\mathrm{Cl}\right.$ I) were as low as $1.5 \times 10^{14}$ $\mathrm{cm}^{-2}$, then the $f\left(\mathrm{H}_{2}\right)$ in zone(s) 1 could be as high as 0.75 - but note the effects of possible differences in depletion discussed in the next paragraph. Some of the H I in zone 2, perhaps of order $2-3 \times 10^{20} \mathrm{~cm}^{-2}$, could be associated with the weak, outlying components seen in Na I (Welty \& Hobbs 2001).

While the model of Jura \& York (1978) assumes that the depletion of $\mathrm{Cl}$ is the same in both zones, that assumption may not be valid, as $\mathrm{Cl}$ appears to be slightly more severely depleted in sightlines with higher mean $n_{\mathrm{H}}$ (e.g., Jenkins et al. 1986). If that assumption is dropped, equation A7 in Jura \& York can be generalized to $f_{1}=f(\mathrm{Cl} \mathrm{I})\left[1+D f_{2}\right]-f\left(\mathrm{H}_{2}\right)$, where $f_{i}=N_{i}(\mathrm{H} \mathrm{I}) / N_{\mathrm{H}}$ is the fraction of the total hydrogen as $\mathrm{H} \mathrm{I}$ in zone $i$ [i.e., $f_{1}+f_{2}$ $\left.+f\left(\mathrm{H}_{2}\right)=1\right]$. The quantity $D=\left(d_{2}-d_{1}\right) / d_{1}$, where $d_{1}$ and $d_{2}$ are the depletions of $\mathrm{Cl}$ in the two zones. Further manipulation of the above equation yields $f_{2}=[1-f(\mathrm{Cl} \mathrm{I})] /[1$ $+D f(\mathrm{Cl})]$. The observed $f(\mathrm{Cl} \mathrm{I})$ and $f\left(\mathrm{H}_{2}\right)$, together with the constraints $0 \leq f_{i} \leq 1-$ $f\left(\mathrm{H}_{2}\right)$, then allow us to place limits on the relative depletions of $\mathrm{Cl}$ in the two zones. As the overall depletion of $\mathrm{Cl}$ toward HD 192639 is very mild, however, in this case the depletions in zones 1 and 2 are likely to be fairly similar. Note that for given values of the total sightline 
column densities, more severe depletions of $\mathrm{Cl}$ in zone(s) 1 [versus those in zone(s) 2] would imply more $\mathrm{H}$ I in zone(s) 1 - and thus a lower $f\left(\mathrm{H}_{2}\right)$ there than if the depletions were equal in the two zones.

In principle, such analyses enable the $\mathrm{H}$ I column densities, the $\mathrm{Cl}$ depletions, and the molecular fractions to be estimated separately for both zones, given only the integrated sightline values. In particular, we may thus be able to better identify and characterize the translucent portions of clouds in a complex line of sight. Obviously, accurate values for $f(\mathrm{Cl} \mathrm{I})$ and $f\left(\mathrm{H}_{2}\right)$ are required; the former would greatly benefit from better $f$-values for the $\mathrm{Cl}$ I lines observable with FUSE. It will be very interesting to examine these issues for other more reddened sightlines. For HD 192639 also note that, independent of incertainties of $f$-values, $f\left(\mathrm{H}_{2}\right) \sim 1.7$ times higher than the integrated line of sight value. Similar analyses may show that the integrated values of $f\left(\mathrm{H}_{2}\right)$ are lower limits to $f$ for the densest clouds along reddened lines of sight.

\section{The High-Velocity Components (HVCs)}

\subsection{Previous Observations}

Three members of Cygnus OB1 association were observed by Phillips et al. (1984) with IUE. Their study showed the existence of high-velocity components in two lines of sight including HD 192639. The average velocity of that gas is consistent with the velocity of the components called HVCs in this paper. Their study showed that the HVCs are seen primarily in highly ionized species such as C IV, Si IV (see Fig. 5), S III or Al III, but lower ionization states from Fe II and Mg II are also detected. Nichols-Bohlin \& Fesen (1993) combined IUE data for 22 stars spanning the OB1 and OB3 associations together with IRAS maps of the entire region in an attempt to identify the HVCs with shells seen in IR emission. Their analysis concluded that the UV absorption lines detected with IUE at high-velocity were pervasive throughout the Cygnus associations and appeared to trace a superbubble encompassing OB1 and OB3. They also found the gas to have more high-velocity structures toward Cyg OB1 than Cyg OB3, indicative of gas specifically associated with Cyg OB1.

\subsection{Ionization}

In the FUSE and STIS data, the HVCs are seen clearly in lines from various singly and doubly ionized species (e.g., C II, N II, Si II, Si III, S II, S III); absorption from N I is 
also present (Fig. 3). The predominance of ionized gas (see below) and the relatively high velocities $\left(-80 \mathrm{~km} \mathrm{~s}^{-1}\right)$ suggest that the HVCs probably originated from the interaction of a shock with gas local to the Cyg OB1 association. Part of that shocked gas has cooled and recombined to yield the nitrogen detected in the STIS data. It is also possible that the gas is photoionized by the nearby stars rather than shock ionized, but the presence of the high velocity components leads us to favor the shock hypothesis. The small structures seen in the Si II* and S II lines at between -70 and $-80 \mathrm{~km} \mathrm{~s}^{-1}$ (Fig. 4) suggest the existence of small scale clumps in the swept-up shell, as suggested by Nichols-Bohlin \& Fesen (1993).

The origin of the shocked gas was discussed by Phillips et al. (1984) based on energy considerations. They concluded that the high-velocity gas could originate either from a supernova or from the combined stellar winds from the hot stars present in Cyg OB1, if the ambient interstellar density is below $0.6 \mathrm{~cm}^{-3}$ in order to satisfy the measured stellar wind power produced by the stars in the association (Abbott 1982). We will shortly revisit that question in $\S 5.4$.

The extended velocity distribution $\left(-70\right.$ to $\left.-30 \mathrm{~km} \mathrm{~s}^{-1}\right)$ of ions typically tracing H II region gas such as N II and N II* or Si II* suggest that large H II regions are present over the entire velocity range. Such H II structures were already mentioned by Lozinskaya et al. (1998a) and Lozinskaya (1998b).

\subsection{Electron density and abundances}

The column densities of various species detected in the HVCs, determined via the AOD method over the interval -100 to $-50 \mathrm{~km} \mathrm{~s}^{-1}$, are listed in Table 6 . The $b$-values of the components seen in the Si II* $1264.7 \AA$ line are of order $2.5 \mathrm{~km} \mathrm{~s}^{-1}$, indicating a maximum temperature of order $10,000 \mathrm{~K}$ for these predominantly ionized HVCs. In such warm, ionized gas, the excited fine structure level of Si II is typically populated primarily via collisions with electrons. The observed ratio $\mathrm{N}\left(\mathrm{Si} \mathrm{II}{ }^{*}\right) / \mathrm{N}(\mathrm{Si} \mathrm{II})$ then implies $n_{e}=1.6_{-0.3}^{+0.7} \mathrm{~cm}^{-3}$ (York \& Kinahan 1979).

The column densities listed in Table 6 can be used to estimate $\mathrm{N}(\mathrm{H} \mathrm{I})$ and $\mathrm{N}(\mathrm{H}$ II) in the HV gas. N I, with ionization potential $14.5 \mathrm{eV}$, traces predominantly neutral gas; N II (and N III) trace ionized gas. While the N II $1083 \AA$ line is strongly saturated, we may use $\mathrm{N}\left(\mathrm{N} \mathrm{II}^{*}\right)$ and the $n_{e}$ derived above to estimate $\mathrm{N}(\mathrm{N}$ II $) \sim 6.2 \times 10^{15} \mathrm{~cm}^{-2}$ (York \& Kinahan 1979). Using the solar abundance of -4.03 dex (Grevesse \& Noels 1993) for N, we obtain 
$\mathrm{N}(\mathrm{H} \mathrm{I}) \sim 6.3 \times 10^{16} \mathrm{~cm}^{-2}$ and $\mathrm{N}(\mathrm{H} \mathrm{II}) \geq 6.9 \times 10^{19} \mathrm{~cm}^{-2}$ - so the $\mathrm{HV}$ gas has a neutral fraction of at most $0.1 \%$. Note, however, that N I could be underabundant due to its ionization when hydrogen is mostly ionized (Jenkins et al. 2000). In that case our estimate of the neutral hydrogen based on neutral nitrogen may only be a lower limit. The ratio $\mathrm{N}(\mathrm{H} \mathrm{II}) / n_{e}$ implies a total thickness of about $14 \mathrm{pc}$ for the $\mathrm{HV}$ gas. If sulfur is not depleted, then in principle we could estimate N(H) from N(S II) + N(S III). Since the S III $1190 \AA$ line is saturated and blended with LV C I absorption, we infer $\mathrm{N}(\mathrm{H})>6 \times 10^{19} \mathrm{~cm}^{-2}$ from N(S II) - consistent with the value obtained from nitrogen. Because of the relatively high $\mathrm{N}(\mathrm{H})$ in the HV components, such lines as Si III $\lambda 1206$, Al II $\lambda 1670$, and Fe III $\lambda 1122$ are strongly saturated making estimates of possible depletions of $\mathrm{Si}, \mathrm{Al}$, and $\mathrm{Fe}$ in the $\mathrm{HV}$ gas very difficult. We, however, report a lower limit on the column density of C III estimated by applying the AOD method in the velocity range considered throughout this analysis $\left([-100,-50] \mathrm{km} \mathrm{s}^{-1}\right)$.

While HV gas at similar velocities has also been observed toward a number of stars in Orion (Cowie, Songaila, \& York 1979; Welty et al. 1999 and in prep.), much more HV gas is present toward HD 192639. For species whose column densities have been measured or inferred in the LV and HV regions, the values toward HD 192639 are typically higher by a factor of order 100. The higher column densities have enabled the detections of both neutral species such as N I (and also Na I) and more highly ionized species such as Si IV and C IV. The strong saturation of lines from a number of other species toward HD 192639, however, makes detailed comparisons of ion ratios difficult. Interestingly, the overall $\mathrm{N}(\mathrm{H} \mathrm{I}) / \mathrm{N}(\mathrm{H}$ II $)$ and $n_{e}$ are fairly similar in the two regions, despite the large differences in $\mathrm{N}(\mathrm{H} \mathrm{II})$.

If the $\mathrm{HV}$ gas comes from a radiative shock, as conjectured earlier, it would be interesting to estimate the amount of energy injected into the ISM in order to produce a total hydrogen column density of $\sim 7 \times 10^{19} \mathrm{~cm}^{-2}$ at a projected velocity of $-80 \mathrm{~km} \mathrm{~s}^{-1}$. To do so, one however needs some knowledge about the geometry of the shocked region. We discuss this in the next section.

\subsection{The Energy Balance}

As mentioned in $\S 5.2$, Abbott (1982) measured the stellar wind power produced by all the stars in the Cyg OB1 association to be about $\sim 1.2 \times 10^{38} \mathrm{erg} \mathrm{s}^{-1}$. If those stars have been sustaining those winds for $4 \times 10^{5}$ years, the energy input from these winds in the ISM is about $1.5 \times 10^{51} \mathrm{erg}$. Nichols-Bohlin \& Fesen (1993) noted weak emissions centered on the Cyg OB1 association $\left(2^{\circ} \times 4.5^{\circ}\right.$ in extent $)$ in the IRAS data. In addition, the IUE data, which 
they re-analyzed, indicated traces of a multi-association superbubble of, at least, $4^{\circ} \times 6^{\circ}$ in extent. If the HVCs originate in the first or the second bubble, the linear dimensions of the bubble would be $(62 \times 140)$ pc or $(126 \times 190)$ pc, respectively. For the purpose of this exercise, we assume the 2 bubbles are spherical and have respective linear radii of $31 \mathrm{pc}$ and $63 \mathrm{pc}$. If we further assume that the total hydrogen in the HVCs is homogeneously distributed in the $31 \mathrm{pc}$ or the $63 \mathrm{pc}$ radius shell, we can estimate the mass contained in the shells using the column density of $\sim 7 \times 10^{19} \mathrm{~cm}^{-2}$, which we derived from the $\mathrm{N}$ and $\mathrm{S}$ analyses in the previous section. We find a mass of $6.7 \times 10^{3} \mathrm{M}_{\odot}$ and $2.8 \times 10^{4} \mathrm{M}_{\odot}$ for the shell with a radius of $31 \mathrm{pc}$ and $63 \mathrm{pc}$, respectively. The corresponding kinetic energies for a projected velocity of $80 \mathrm{~km} \mathrm{~s}^{-1}$ are $4.2 \times 10^{50} \mathrm{erg}$ and $1.8 \times 10^{51} \mathrm{erg}$ for the $2^{\circ}$ and the $4^{\circ}$ shells respectively. Are the stellar winds in Cyg OB1 capable of producing such energy? How does it compare with supernova energy injection? The model of spherical wind bubble developed by Weaver et al. (1977), with the above-mentioned radius, HV gas velocity and stellar wind energy $\left(1.5 \times 10^{51} \mathrm{erg}\right)$ derived from Abbott (1982), indicates that the ambient pre-shock density has to be lower than $0.6 \mathrm{~cm}^{-3}$. If we assume this value to be our upper limit on the ambient density in that region, the corresponding upper limits on the energy produced by a supernova (Chevalier 1974) are $6.2 \times 10^{50} \mathrm{erg}(31 \mathrm{pc})$ and $5.7 \times 10^{51} \mathrm{erg}(63 \mathrm{pc})$. The comparison of those estimates with the kinetic energies required to produce the hydrogen shells considered above, indicates that the distinction between a stellar wind or a supernova origin cannot be made on energy considerations only, under our assumptions. Lozinskaya (1998b) explained the multi-shell structure seen toward Cyg OB1 and OB3, as the result of a 2-step kinematics in which the winds from the OB stars create shells of low-velocity gas with which the winds of WR stars (or supernovae appearing later in the association's history) interact, forming higher-velocity shells. A study of the HV gas ionization structure would be required to probe their conclusions. This study will be presented in a separate work.

\section{Summary/Perspectives}

We have presented here a survey of the detailed analyses that can be performed when using data from different instruments. We combined the far-UV dataset from FUSE, HST/STIS and $I U E$ archival datasets with high-resolution optical data and focused on the study of the abundances and physical conditions of the low-velocity interstellar gas toward the candidate translucent cloud star HD 192639. A brief overview of the high-velocity gas components seen along this line of sight was also given. The high-resolution optical K I data were used to define the velocity structure in the neutral gas that could not be resolved with the FUSE data $\left(18 \mathrm{~km} \mathrm{~s}^{-1}\right)$ and could not be entirely resolved with the STIS data $\left(2.75 \mathrm{~km} \mathrm{~s}^{-1}\right)$. We did not use a specific velocity structure model when analyzing the highly-ionized gas. 
The neutral gas exhibits multiple low-velocity components (LVCs) at velocities between -18 and $-8 \mathrm{~km} \mathrm{~s}^{-1}$ (heliocentric). It consists of gas with temperatures and densities typical of cold diffuse clouds mixed with slightly warmer gas. The analysis of the $\mathrm{H}_{2} J=0$ and 1 transitions indicates a temperature of that gas of $(90 \pm 10) \mathrm{K}$. The higher rotational transitions $(J=2)$ seem to be in thermal equilibrium at $\mathrm{T}_{01}$. The trace neutral elements $\mathrm{C} \mathrm{I}$ and S I show profiles very similar to that of K I, suggesting their co-location in the LV gas. Analysis of the C I fine-structure excitation led to an estimate of the line-of-sight neutral hydrogen density of $(16 \pm 3) \mathrm{cm}^{-3}$, typical for cold diffuse clouds. The detection of $\mathrm{O} \mathrm{I}^{*}$ and O I** at the velocity of the LVCs, suggests densities very similar to those derived from the carbon analysis, ranging from 11 to $26 \mathrm{~cm}^{-3}$. The depletion study shows that the average properties of the LV gas toward HD 192639 are close to those exhibited by diffuse clouds rather than by translucent clouds (Snow 1975; Snow et al. 2000; Rachford et al. 2001a). The line of sight seems dominated by multiple diffuse clouds, as already indicated by the velocity structure and supported by the component-by-component analysis of the partially resolved STIS data. Analysis of the overall $\mathrm{Cl}$ I, Cl II, and $\mathrm{f}\left(\mathrm{H}_{2}\right)$ indicates higher molecular fractions in the regions where $\mathrm{H}_{2}$ is dominant and confirms the diffuse nature of the cold $\mathrm{LV}$ gas. The average electron density, derived from five pairs of elements, is estimated to be $(0.11 \pm 0.02)$ $\mathrm{cm}^{-3}$ - leading to an ionization fraction in the LVCs of 0.007, much larger than expected from carbon ionization alone. The LVCs seem therefore to contain some ionized hydrogen in this predominantly neutral material. Alternatively, the high fractional ionization could be partly due to an overestimation of $n_{e}$ if the ionization equilibrium is affected by other processes besides photoionization and radiative recombination.

Our overview study of the high velocity gas shows that the latter is also made of multiple blended components (HVCs) ranging in velocity from -60 to $-80 \mathrm{~km} \mathrm{~s}^{-1}$ (heliocentric). This gas is predominantly ionized, but also contains a small amount in neutral form at the same velocity, suggesting fast cooling and recombination. Some of the material may be due to a radiative shock. The silicon fine-structure excitation analysis suggests that the $\mathrm{H}$ II region has an electron density of the order of $1.6 \mathrm{~cm}^{-3}$. A lower limit estimate to the total hydrogen column density of $\sim 7 \times 10^{19} \mathrm{~cm}^{-2}$ was derived from the nitrogen and sulfur measurements in the HV gas. The kinetic energy injected into the ISM to generate the measured hydrogen column density at the HV gas velocity can be produced by either stellar winds or supernovae.

Complementary HST data would be useful to get Mg I, Fe I, S II, Si I, Si II and Zn II in order to derive, directly, the electron densities and further study the depletions in the 
LV gas. Further observations of Cyg OB1 stars located in a $3^{\circ}$ radius of HD 192639 would provide unique insights in the small-scale density (and pressure) variations of the ISM as seen toward HD 192639. SNR shocks show further signatures in the optical in the form of characteristic emission lines such as those from [O III], [S II] or [N II]. Optical observations of HD 192639 performed offset from the star itself would allow a search for those emission lines and provide a test for the supernova remnant shock origin of the HVCs toward HD 192639. A study of the Cl I $f$-values in the FUSE bandpass is warranted.

This work is based on data obtained for the Guaranteed Time Team by the NASACNES-CSA FUSE mission operated by the Johns Hopkins University. Financial support to U. S. participants has been provided by NASA contract NAS5-32985. D. E. Welty acknowledges support from the NASA LSTA grant NAG5-3228 to the University of Chicago. This

work has been done using the profile fitting procedure Owens.f developed by M. Lemoine and the FUSE French Team. This research has made use of the SIMBAD database, operated at CDS, Strasbourg, France. The authors thank M. André for fruitful comments on the FUSE data reduction.

\section{REFERENCES}

Abbott, D. C. 1982, ApJ, 263, 723

Aldrovandi, S. M. V., \& Péquignot, D. 1973, A\&A, 25, 137

Aldrovandi, S. M. V., \& Péquignot, D. 1974, Rev. Brasiliera de Fisica, 4, 491

Anders, E., \& Grevesse, N. 1989, Geochim. Cosmochim. Acta, 53, 197

Beideck, D. J., Schectman, R. M., Federman, S. R., \& Ellis, D. G. 1994, ApJ, 428, 393

Black, J. H., \& Dalgarno, A. 1973, ApJ, 184, L101

Chevalier, R. A. 1974, ApJ, 188, 501

Cowie, L. L., Songaila, A., \& York, D. G. 1979, ApJ, 230, 469

Danks, A. C., Federman, S. R., \& Lambert, D. L. 1984, A\&A, 130, 62

Diplas, A., \& Savage, D. B. 1994, ApJ,93, 274

Draine, B. T. 1978, ApJS, 36, 595 
Federman, S. R., Fritts, M., Cheng, S., Menningen, K. M., Knauth, D. C., \& Fulk , K. 2001, ApJS, 134, 133

Grevesse, N., \& Noels, A. 1993, in Origin of the Elements, ed. N. Prantzos et al. (Cambridge: Cambridge Univ. Press), 15

Holweger, H. 2001, Solar and Galactic Composition, ed. R. F. Wimmer-Schweingruber (Berlin: Springer), in press

Howk, J. C., Sembach, R. K., Roth, K. C., \& Kruk, J. W. 2000, ApJ, 544, 867

Howk, J. C., \& Sembach, R. K. 2000, ApJ, 119, 2481

Jenkins, E. B., \& Shaya, E. J. 1979, ApJ, 231, 55

Jenkins, E. B., Savage, B. D., \& Spitzer, L. 1986, ApJ, 301, 355

Jenkins, E. B., \& Peimbert, A. 1997, ApJ, 477, 265

Jenkins, E. B., et al. 2000, ApJ, 538, L81

Jenkins, E. B., \& Tripp, T. M. 2001, ApJS, 137, 297

Jura, M. 1974, ApJ, 190, 33

Jura, M., \& York, D. G. 1978, ApJ, 219, 861

Keenan, F. P., \& Berrington, K. A. 1988, ApJ, 333, 806

Kruk, J. W., \& Murphy, E. 2001 "The CalFUSE Pipeline Reference Guide," http://fuse.pha.jhu.edu/analysis/pipeline-reference-v0.6.html

Lemoine, M. et al. 2001, ApJS, in press

Lepp, S., Dalgarno, A., van Dishoeck, E. F., \& Black, J. H. 1988, ApJ, 329, 418

Lozinskaya, T. A., Pravdikova, V. V., Sitnik, T. G., Episov, V. F., \& Mel'nikov, V. V. 1998a, Astronomy Reports, 42, 514

Lozinskaya, T. A. 1998b, Astronomy Letters, Vol. 24, No 2, 237

Moos, H. W., et al. 2000, ApJ, 538, L1

Morton, D. C. 1991, ApJS, 77, 119

Morton, D. C., \& Noreau, L. 1994, ApJS, 95, 301 
Morton, D. C. 2001, in preparation

Nichols-Bohlin, J., \& Fesen, R. A. 1993, AJ, 105, 672

Péquignot, D., \& Aldrovandi, S. M. V. 1986, A\&A, 161, 169

Phillips, A. P., Welsh, B. Y., \& Pettini, M. 1984, MNRAS, 206, 55

Rachford, B. L., Snow, T. P., Tumlinson, J., Shull, J. M., Roueff, E., André, M., Desert, J.-M., Ferlet, R., Vidal-Madjar, A., \& York, D. G. 2001a, ApJ, 555, 839

Rachford, B. L. et al. 2001b, submitted

Sahnow, D. J. et al. 2000, ApJ, 538, L7

Schectman, R. M., Federman, S. R., Beideck, D. J., \& Ellis, D. G. 1993, ApJ, 406, 735

Shull, J. M., \& van Steenberg, M. 1982, ApJS, 48, 95

Snow, T. P. 1975 , ApJ, 202, 87

Snow, T. P. et al. 2000, ApJ, 538, L65

Sofia, U. J., Cardelli, J. A., Guerin, K. P., \& Meyer, D. M. 1997, ApJ, 482, L105

Sofia, U. J., Fabian, D., \& Howk, J. C. 2000, ApJ, 531, 384

Spitzer, L., \& Fitzpatrick, E. L. 1993, ApJ, 409, 299

St-Louis, N., \& Smith, L. J. 1991, A\&A, 252, 781

Walborn, N. R. 1972, AJ, 77, 312

Weaver, R., McCray, R., Castor, J., Shapiro, P., \& Moore, R. 1977, 218, 377

Weingartner, J. C., \& Draine, B. T. 2001, ApJ, 563, 842

Welty, D. E., Hobbs, L. M., Lauroesch, J. T., Morton, D. C., Spitzer, L., \& York, D. C. 1999, ApJS, 124, 465

Welty, D. E., \& Hobbs, L. M. 2001, ApJS, 133, 345

Welty, D. E., Hobbs, L. M., \& Morton, D. C., 2002a, ApJ, submitted

Welty, D. E., Snow, T. P., \& Morton, D. C., 2002b, in preparation

Witt, A. N., \& Johnson, M. W. 1973, ApJ, 181, 363 
York, D. G., \& Kinahan, B. F. 1979, ApJ, 228, 127 
Fig. 1.- The upper left panel contains a sample of $\mathrm{H}_{2}$ lines from the LVCs detected in the FUSE LiF1A spectrum. The low $J$ lines are labeled, correspondingly in wavelength space. The higher $J$ levels seen in this panel are labeled from 1 to 5 and represented in each of the 5 remaining panels using heliocentric velocities. The tick marks indicate the position of the 5 components forming the LVCs.
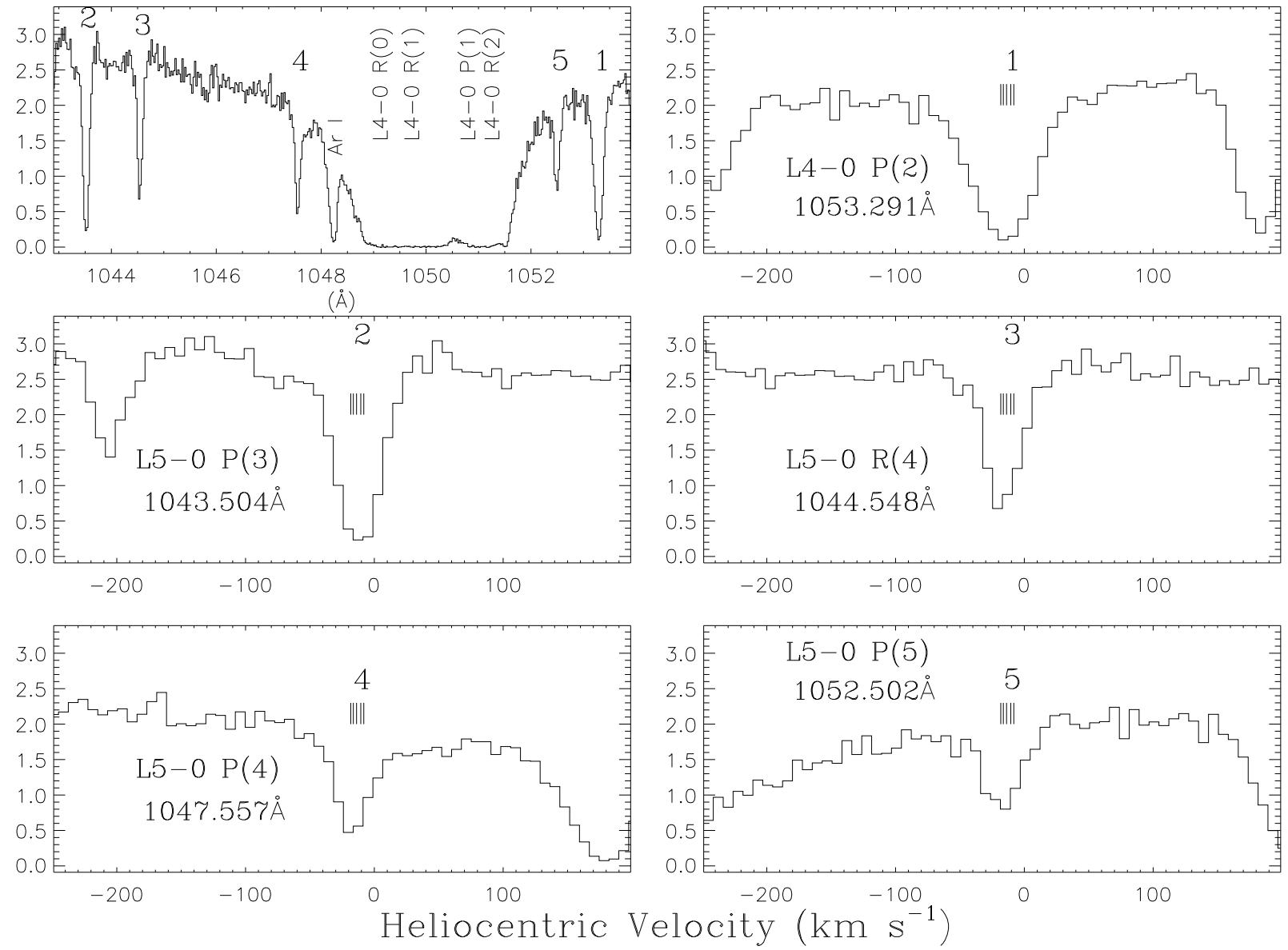
Fig. 2.- Absorption from the trace neutral species in the LVCs. The wavelengths correspond to the element indicated by the tick marks. The source of the data is indicated at the bottom of each panel: (I) for IUE data, (F) for FUSE data, (O) for optical data and (S) for STIS data. The tick marks indicate the position of the 5 components forming the LVCs.
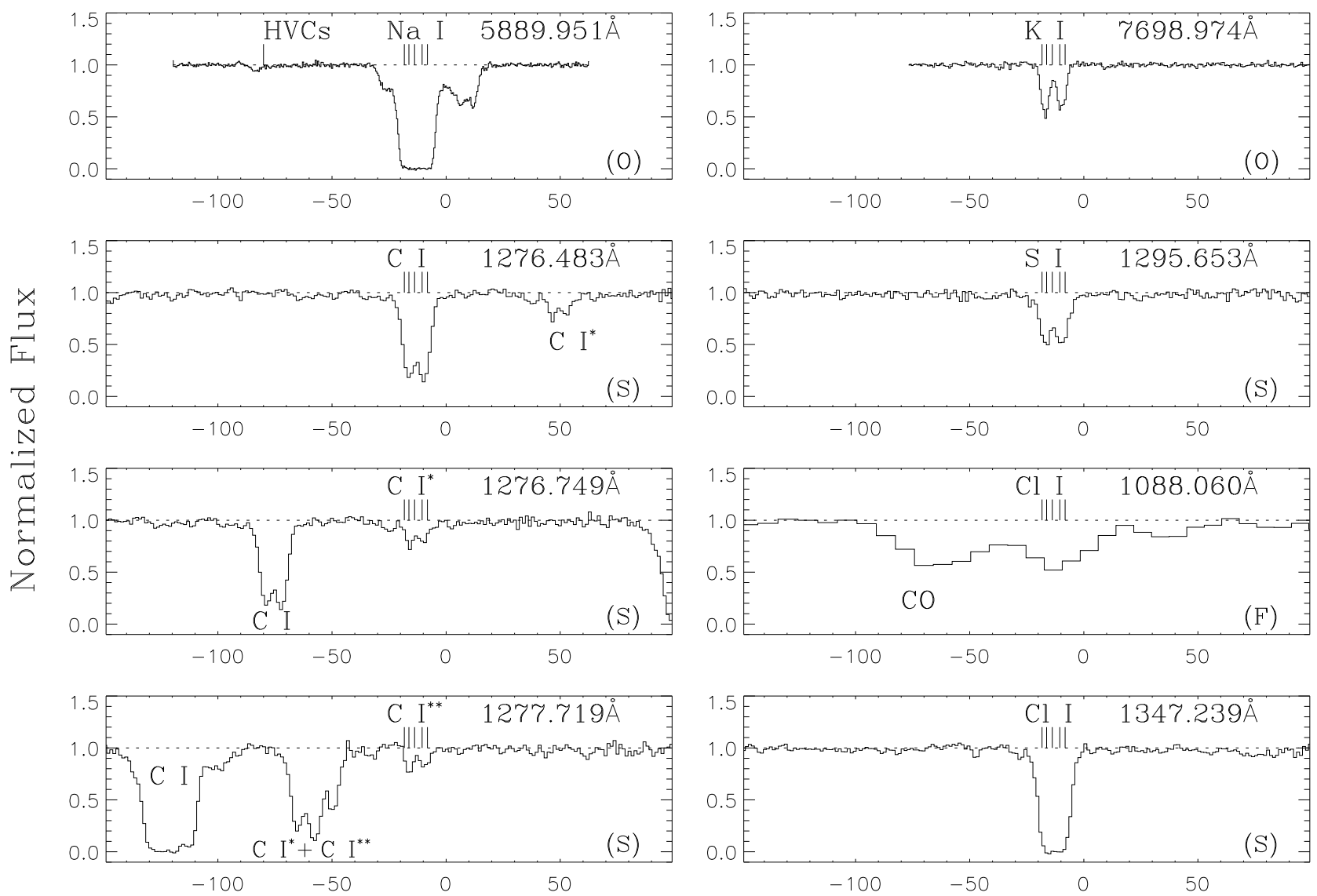

Heliocentric Velocity $\left(\mathrm{km} \mathrm{s}^{-1}\right)$ 
Fig. 3.- Absorption from C II, N I and O I in the LVCs and the HVCs.
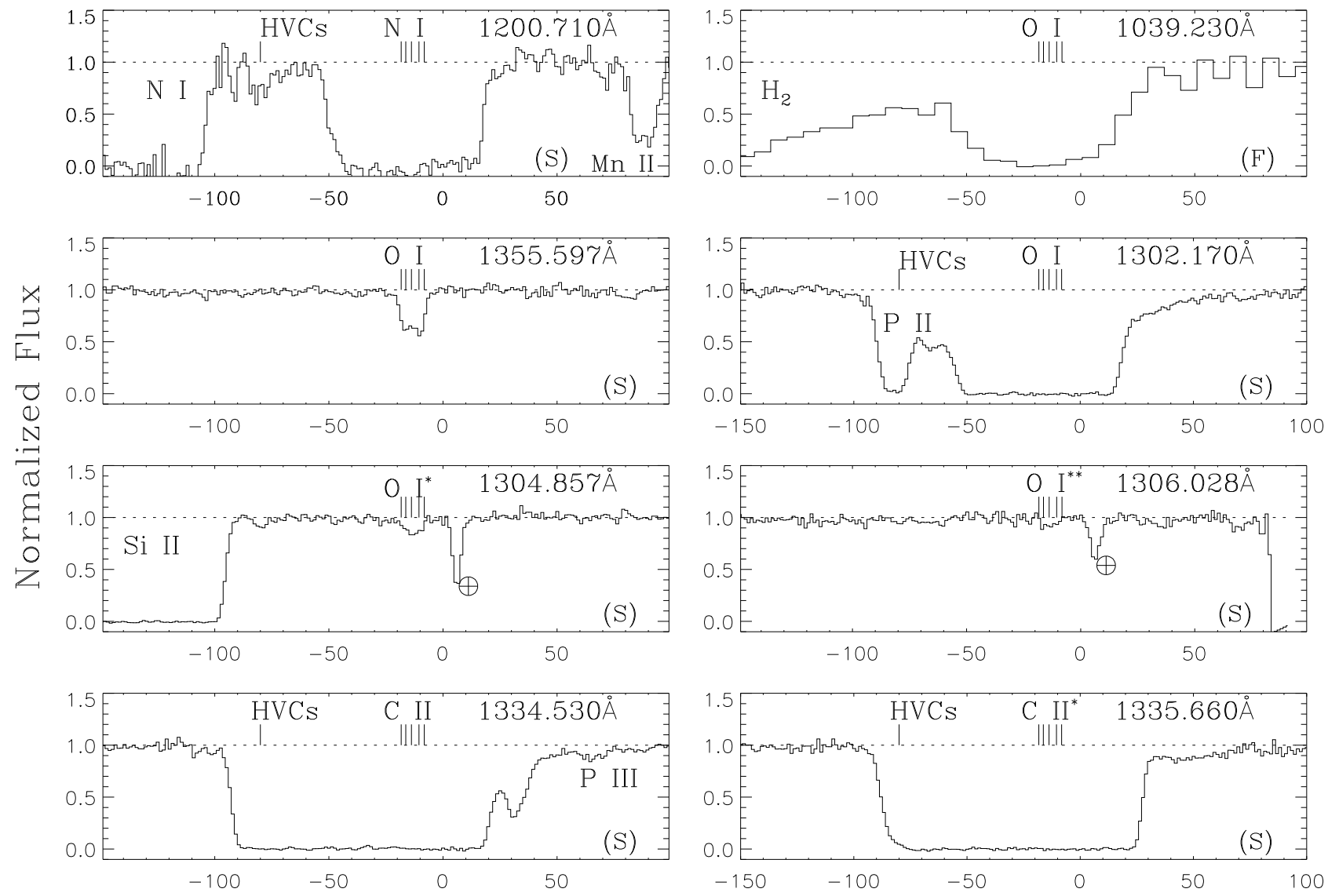

Heliocentric Velocity $\left(\mathrm{km} \mathrm{s}^{-1}\right)$ 
Fig. 4.- Absorption from singly ionized species in the LVCs and the HVCs.
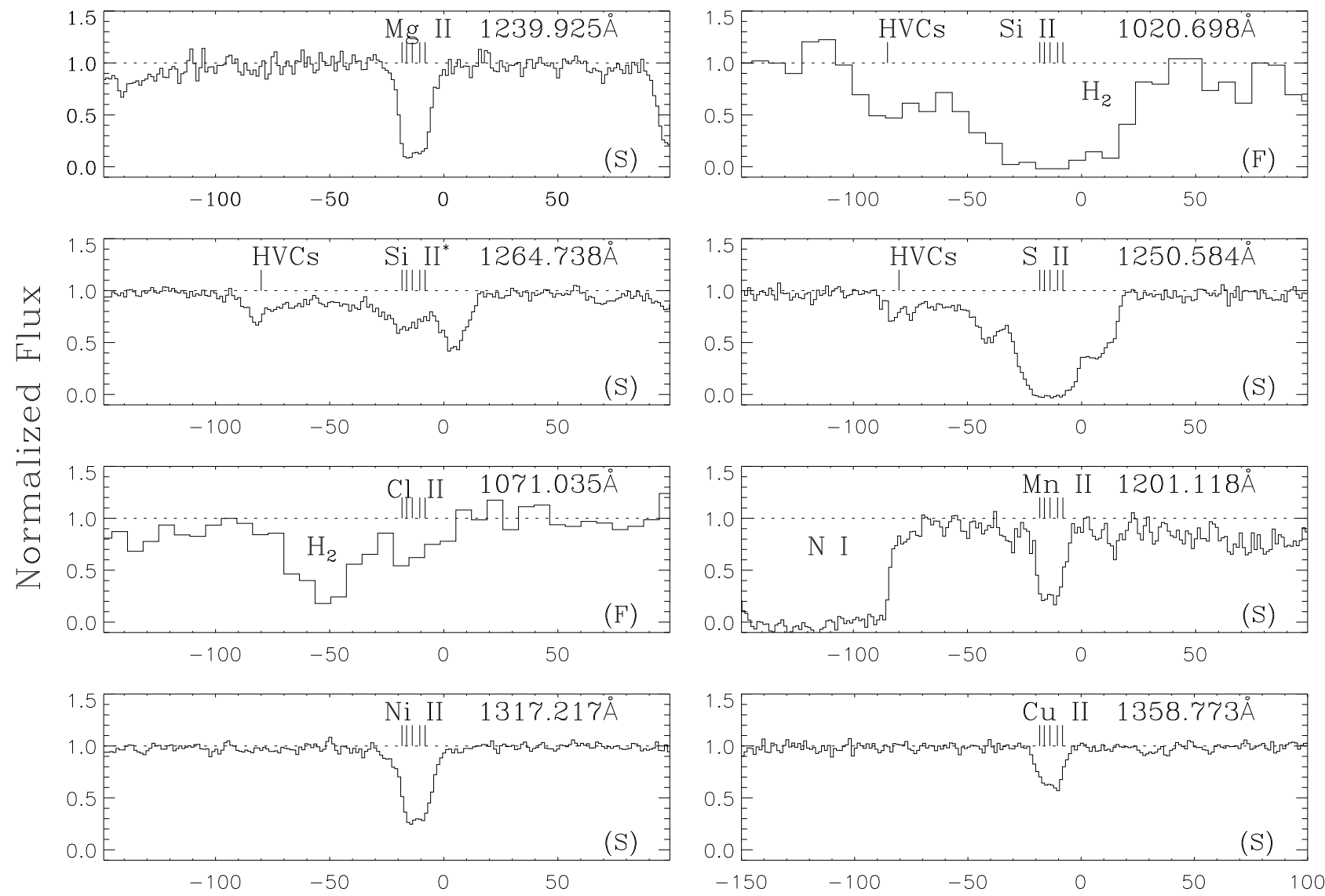

Heliocentric Velocity $\left(\mathrm{km} \mathrm{s}^{-1}\right)$ 
Fig. 5.- Strong lines from ionized species clearly delineate the HV gas.
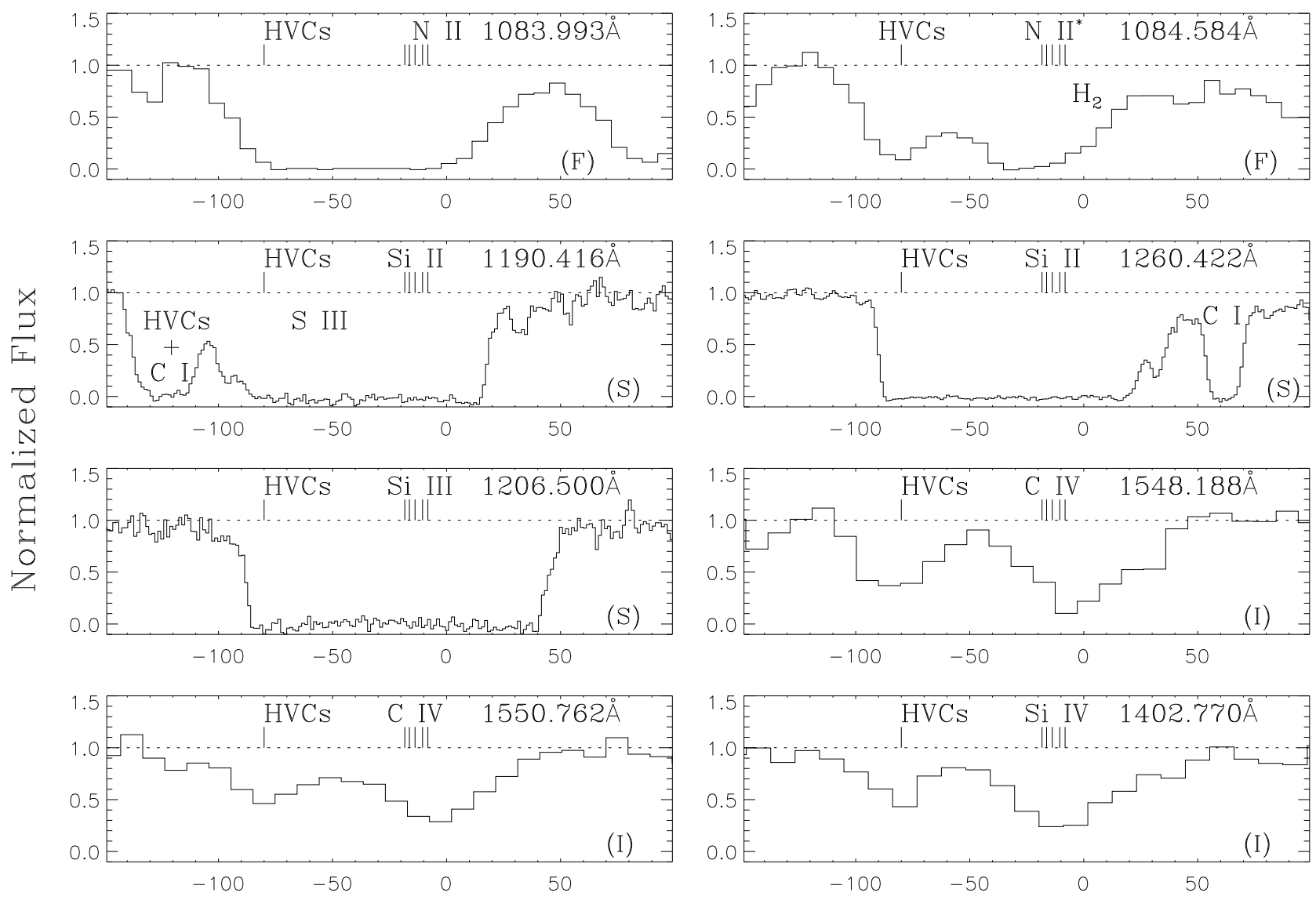

Heliocentric Velocity $\left(\mathrm{km} \mathrm{s}^{-1}\right)$ 
Fig. 6.- Comparison of the O I, O I* and O I** profiles in the LV gas. The ' $\oplus$ ' symbol denotes the position of the telluric lines. The tick marks indicate the position of the 5 components forming the LVCs.

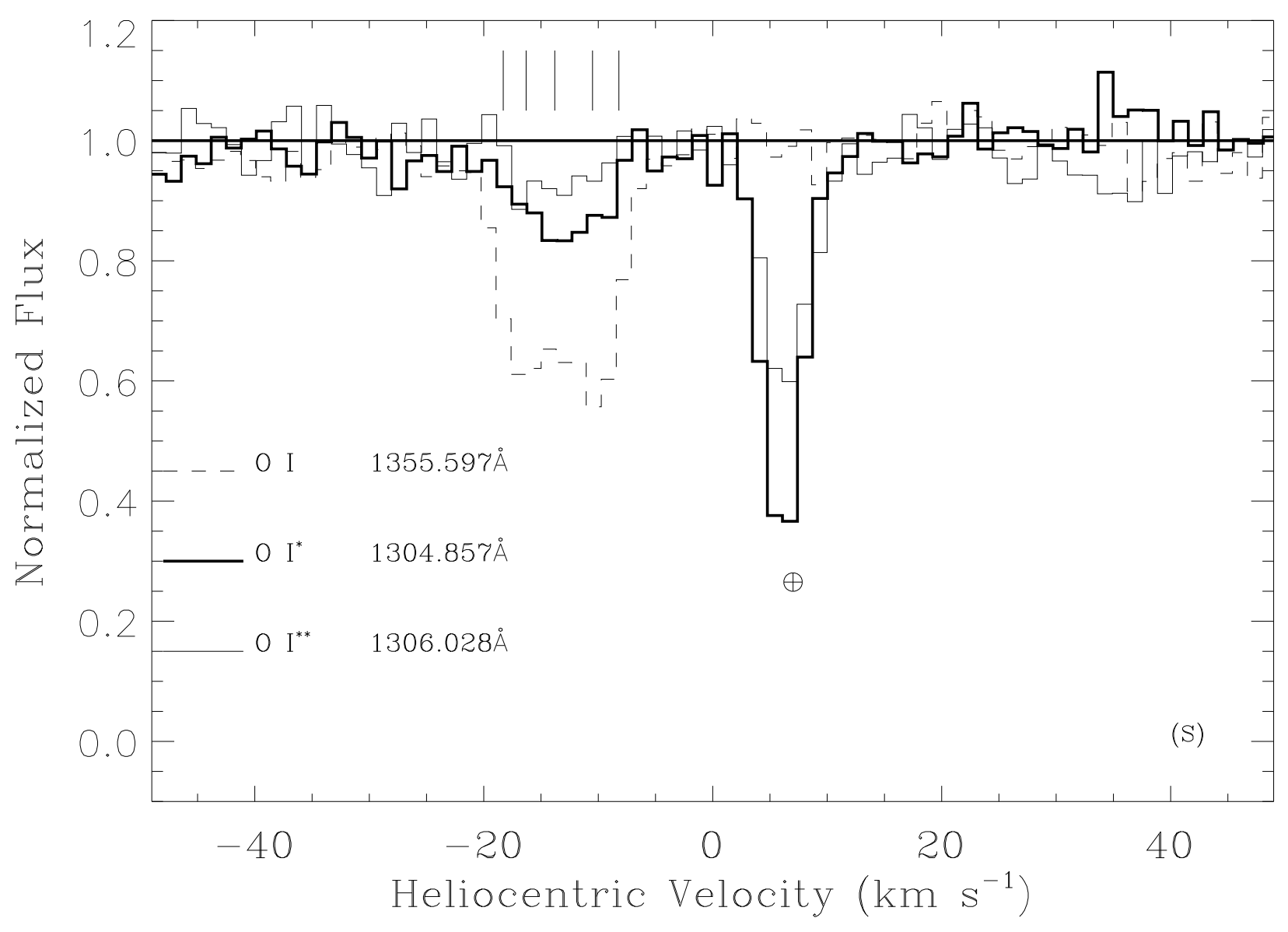


Fig. 7.- Curve of growth for C I, C I* and C I** detected with FUSE and HST/STIS.
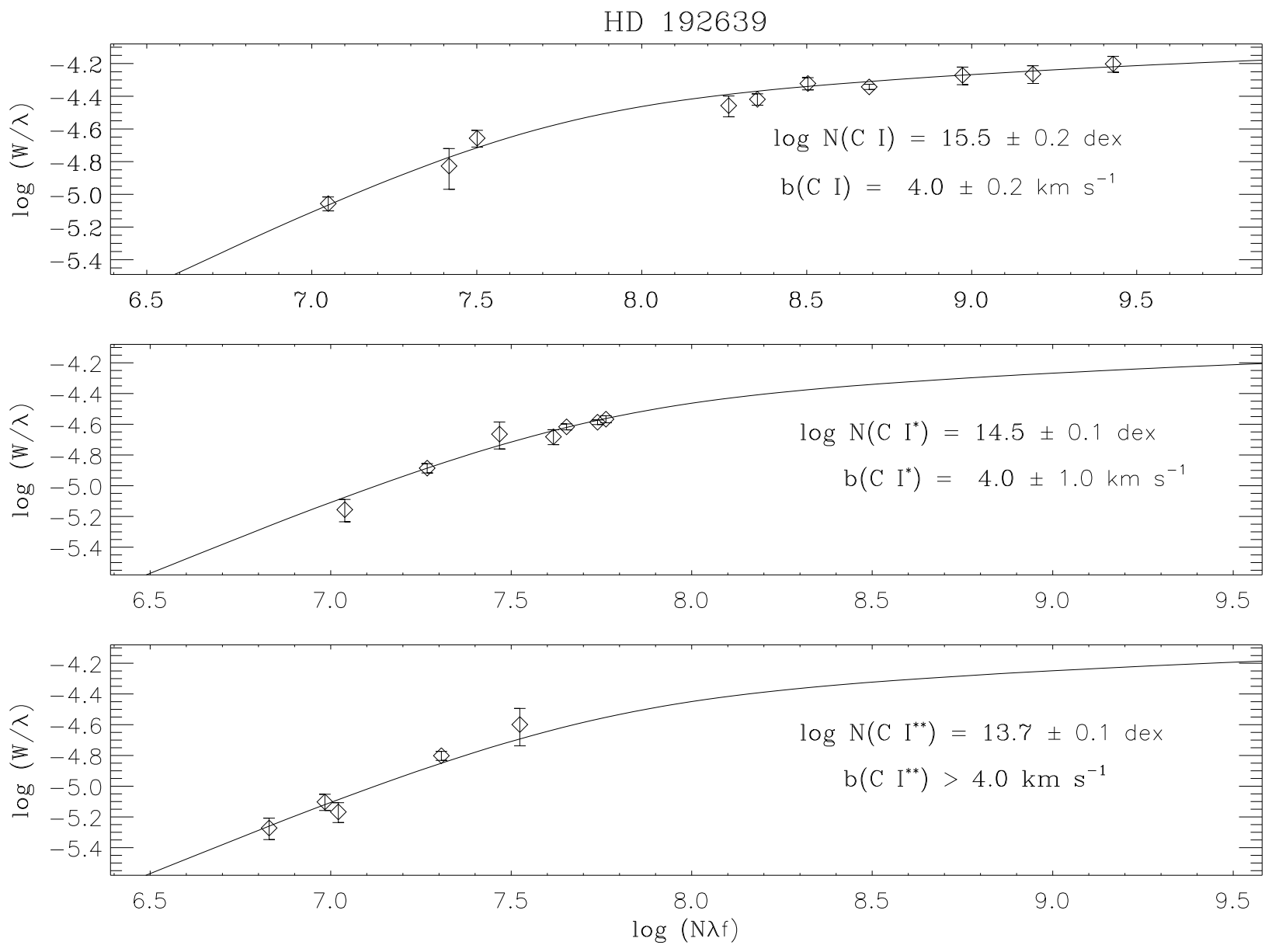
Fig. 8.- Comparison of the gas-phase depletions in the LVCs and the depletions from the "typical" cold and warm interstellar gas (Welty et al. 1999) toward HD 192639.

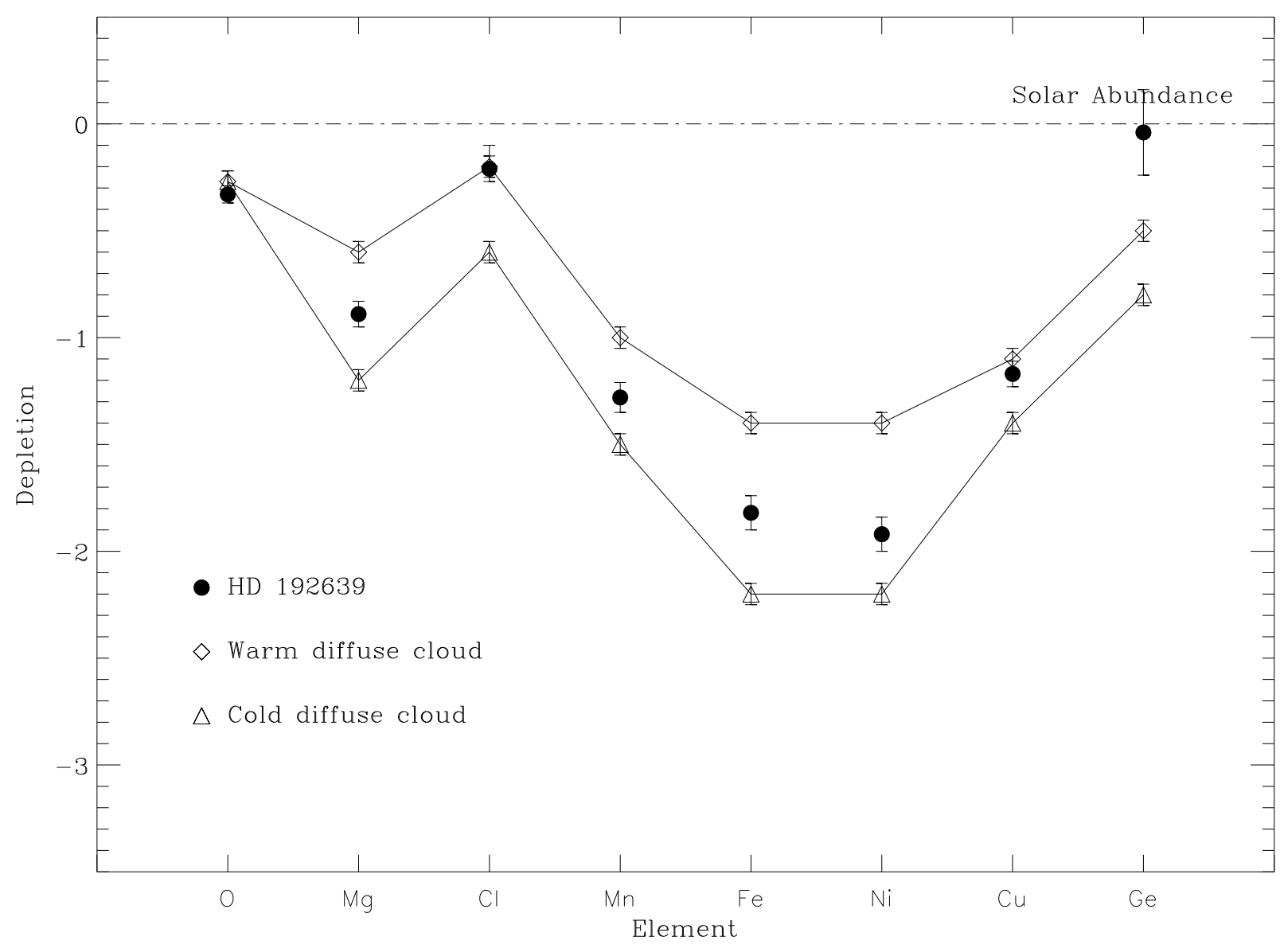


Table 1. Total Equivalent Widths in the LVCs toward HD 192639.

\begin{tabular}{lcccc}
\hline \hline Species & $\lambda^{a}$ & $\log (f \lambda)^{a}$ & $\begin{array}{c}W \\
(\mathrm{~m} \AA)\end{array}$ & Dataset \\
& $(\AA)$ & & & \\
\hline C I & 1129.195 & 0.998 & $53.8 \pm 4.6$ & FUSE \\
& 1129.317 & $-0.089^{b}$ & $16.8 \pm 4.7$ & FUSE \\
& 1155.809 & 0.845 & $44.1 \pm 3.5$ & FUSE \\
& 1192.215 & -0.004 & $26.3 \pm 3.1$ & STIS \\
& 1193.995 & 1.184 & $54.0 \pm 2.0$ & STIS \\
& 1260.735 & 1.681 & $68.6 \pm 8.5$ & STIS \\
& 1270.143 & -0.455 & $11.2 \pm 1.1$ & STIS \\
& 1276.482 & 0.758 & $44.6 \pm 6.5$ & STIS \\
& 1280.135 & 1.468 & $68.4 \pm 8.4$ & STIS \\
& 1328.833 & 1.924 & $83.5 \pm 9.3$ & STIS \\
C I* & 1193.678 & 1.085 & $24.9 \pm 2.8$ & STIS \\
& 1260.926 & 1.231 & $34.2 \pm 1.8$ & STIS \\
& 1260.996 & 1.122 & $30.5 \pm 1.4$ & STIS \\
P III & 1334.813 & 1.576 & $5.4 \pm 1.7$ & STIS
\end{tabular}


Table 1-Continued

\begin{tabular}{lcclr}
\hline \hline Species & $\begin{array}{c}\lambda^{a} \\
(\AA)\end{array}$ & $\log (f \lambda)^{a}$ & \multicolumn{1}{c}{$W$} & Dataset \\
& $(\mathrm{m} \AA)$ & \\
\hline S I & 1295.653 & $2.052^{d}$ & $27.0 \pm 1.0$ & STIS \\
& 1296.174 & $1.455^{d}$ & $9.0 \pm 1.0$ & STIS \\
Cl I & 1004.677 & 2.200 & $62.0 \pm 14.0$ & FUSE \\
& 1088.059 & $1.945^{e}$ & $50.0 \pm 15.0$ & FUSE \\
& 1097.370 & $0.985^{e}$ & $10.0 \pm 3.0$ & FUSE \\
& 1347.239 & $2.314^{e}$ & $70.0 \pm 2.0$ & STIS \\
Cl II & 1071.035 & 1.206 & $26.3 \pm 7.4$ & FUSE \\
Ar I & 1048.224 & 2.440 & $>175$ & FUSE \\
Mn II & 1197.184 & 2.415 & $52.5 \pm 3.2$ & STIS \\
& 1201.118 & 2.163 & $39.4 \pm 2.4$ & STIS \\
Fe II & 1055.261 & $0.898^{f}$ & $44.3 \pm 8.9$ & FUSE \\
& 1112.048 & $0.839^{f}$ & $46.8 \pm 6.6$ & FUSE \\
& 1121.975 & $1.355^{f}$ & $93.2 \pm 5.9$ & FUSE \\
& 1125.447 & $1.255^{f}$ & $92.9 \pm 8.3$ & FUSE \\
& 1127.098 & $0.499^{f}$ & $39.5 \pm 7.2$ & FUSE \\
& 1133.665 & $0.795^{f}$ & $75.0 \pm 6.0$ & FUSE \\
& 1142.365 & $0.681^{f}$ & $47.5 \pm 4.6$ & FUSE \\
& 1143.226 & $1.306^{f}$ & $85.8 \pm 5.4$ & FUSE \\
& 1144.938 & $2.084^{f}$ & $137.0 \pm 5.0$ & FUSE \\
Ni II & 1317.217 & $2.009^{g}$ & $49.0 \pm 1.0$ & STIS \\
Cu II & $1358.773^{b}$ & $2.713^{b}$ & $19.9 \pm 1.0$ & STIS \\
Ge II & $1237.059^{g}$ & $3.183^{g}$ & $44.9 \pm 3.8$ & STIS \\
CO & $1076.034^{h}$ & $1.864^{h}$ & $48.0 \pm 9.2$ & FUSE \\
& $1087.868^{i}$ & $2.107^{i}$ & $55.0 \pm 16.0$ & FUSE \\
& $1344.186^{i}$ & $0.745^{i}$ & $5.45 \pm 2.1$ & STIS \\
\hline & & & & \\
& & & &
\end{tabular}

a Vacuum wavelengths and $f$-values from Morton (2001), unless noted otherwise: (b) Morton (1991); (c) Sofia et al. (2000); (d) Beideck et al. (1994); (e) Schectman et al. (1993); (f) Howk et al. (2000); (g) Welty et al. (1999); (h) Federman et al. (2001) and (i) Morton \& Noreau (1994). Uncertainties are $1 \sigma$. 
Table 2. Column Densities of the individual Components of the LVCs.

\begin{tabular}{|c|c|c|c|c|c|}
\hline $\begin{array}{c}\text { Species } \\
v\left(\mathrm{~km} \mathrm{~s}^{-1}\right) \\
b\left(\mathrm{~km} \mathrm{~s}^{-1}\right)\end{array}$ & $\begin{array}{c}\text { Comp } 1 \\
-18.5 \\
0.8\end{array}$ & $\begin{array}{c}\text { Comp } 2 \\
-16.5 \\
1.1\end{array}$ & $\begin{array}{c}\text { Comp } 3 \\
-14.0 \\
1.0\end{array}$ & $\begin{array}{c}\text { Comp } 4 \\
-10.7 \\
1.2\end{array}$ & $\begin{array}{c}\text { Comp } 5 \\
-8.4 \\
1.1\end{array}$ \\
\hline C I & $5.5 \pm 5.0 \mathrm{E} 13$ & $6.5 \pm 1.6 \mathrm{E} 14$ & $2.7 \pm 0.9 \mathrm{E} 14$ & $8.5 \pm 5.9 \mathrm{E} 14$ & $1.4 \pm 0.5 \mathrm{E} 14$ \\
\hline C I* & $1.1 \pm 1.0 \mathrm{E} 13$ & $1.5 \pm 1.1 \mathrm{E} 14$ & $4.3 \pm 2.1 \mathrm{E} 13$ & $1.1 \pm 1.0 \mathrm{E} 14$ & $2.5 \pm 0.8 \mathrm{E} 13$ \\
\hline C I** & $1.3 \pm 1.0 \mathrm{E} 12$ & $1.9 \pm 1.2 \mathrm{E} 13$ & $6.1 \pm 2.9 \mathrm{E} 12$ & $1.3 \pm 0.6 \mathrm{E} 13$ & $4.9 \pm 2.3 \mathrm{E} 12$ \\
\hline O I & $2.4 \pm 1.5 \mathrm{E} 17$ & $2.7 \pm 1.5 \mathrm{E} 17$ & $2.9 \pm 0.2 \mathrm{E} 17$ & $3.5 \pm 0.3 \mathrm{E} 17$ & $2.3 \pm 1.6 \mathrm{E} 17$ \\
\hline O I* & $5.3 \pm 2.3 \mathrm{E} 11$ & $1.4 \pm 0.2 \mathrm{E} 12$ & $2.8 \pm 0.4 \mathrm{E} 12$ & $2.2 \pm 0.1 \mathrm{E} 12$ & $<5.2 \mathrm{E} 10$ \\
\hline O I** & - & $2.1 \pm 0.6 \mathrm{E} 12$ & $1.3 \pm 1.0 \mathrm{E} 12$ & $1.2 \pm 0.2 \mathrm{E} 12$ & - \\
\hline $\mathrm{Na} \mathrm{I}^{a}$ & [1.01E13] & [2.99E13] & {$[6.10 \mathrm{E} 12]$} & [1.99E13] & [1.44E13] \\
\hline Mg II & $2.2 \pm 1.7 \mathrm{E} 15$ & $5.5 \pm 0.2 \mathrm{E} 15$ & $4.4 \pm 3.5 \mathrm{E} 15$ & $3.9 \pm 3.6 \mathrm{E} 15$ & $3.4 \pm 1.1 \mathrm{E} 15$ \\
\hline S I & $5.1 \pm 1.3 \mathrm{E} 12$ & $8.5 \pm 1.2 \mathrm{E} 12$ & $4.7 \pm 2.2 \mathrm{E} 12$ & $7.1 \pm 1.0 \mathrm{E} 12$ & $3.2 \pm 1.5 \mathrm{E} 12$ \\
\hline K I & $1.0 \pm 0.1 \mathrm{E} 11$ & $2.5 \pm 0.1 \mathrm{E} 11$ & $0.6 \pm 0.1 \mathrm{E} 11$ & $2.0 \pm 0.2 \mathrm{E} 11$ & $1.4 \pm 0.2 \mathrm{E} 11$ \\
\hline Mn II & $8.9 \pm 0.8 \mathrm{E} 12$ & $1.5 \pm 0.5 \mathrm{E} 13$ & $1.6 \pm 0.2 \mathrm{E} 13$ & $2.7 \pm 0.3 \mathrm{E} 13$ & $4.7 \pm 0.2 \mathrm{E} 12$ \\
\hline Ni II & $1.3 \pm 1.1 \mathrm{E} 13$ & $2.1 \pm 0.5 \mathrm{E} 13$ & $1.4 \pm 1.2 \mathrm{E} 13$ & $1.3 \pm 1.2 \mathrm{E} 13$ & $9.3 \pm 0.4 \mathrm{E} 12$ \\
\hline $\mathrm{Cu}$ II & $2.7 \pm 1.4 \mathrm{E} 11$ & $1.1 \pm 0.3 \mathrm{E} 12$ & $1.3 \pm 0.3 \mathrm{E} 12$ & $1.7 \pm 0.9 \mathrm{E} 12$ & $6.7 \pm 3.5 \mathrm{E} 11$ \\
\hline Ge II & $8.7 \pm 2.1 \mathrm{E} 11$ & $2.1 \pm 1.5 \mathrm{E} 12$ & $2.7 \pm 1.2 \mathrm{E} 11$ & $1.7 \pm 0.7 \mathrm{E} 13$ & - \\
\hline
\end{tabular}

\footnotetext{
a Values in brackets were fixed in the fitting. Uncertainties are $1 \sigma$. Column densities are given in $\mathrm{cm}^{-2} . v$ and $b$ are the velocity and $b$-value for each component, respectively.
} 
Table 3. Column Densities in the LVCs toward HD 192639.

\begin{tabular}{|c|c|c|c|}
\hline Species & $\begin{array}{l}\log N \\
(\operatorname{dex})\end{array}$ & Methods $^{a}$ & Dataset \\
\hline $\mathrm{C}$ I & $15.29 \pm 0.08$ & COG;AOD;FIT & FUSE+STIS \\
\hline $\mathrm{CI}^{*}$ & $14.53 \pm 0.08$ & COG;AOD;FIT & FUSE+STIS \\
\hline $\mathrm{C} \mathrm{I}^{* *}$ & $13.67 \pm 0.08$ & COG;AOD;FIT & FUSE+STIS \\
\hline O I & $18.16 \pm 0.11$ & AOD;COG;FIT & FUSE+STIS \\
\hline O I* & $12.91 \pm 0.10$ & EW;FIT & STIS \\
\hline $\mathrm{O} \mathrm{I}^{* *}$ & $12.73 \pm 0.15$ & EW;FIT & STIS \\
\hline $\mathrm{Na} \mathrm{I}$ & $13.91 \pm 0.04$ & FIT & Optical \\
\hline Mg I & $>14.09$ & AOD & IUE \\
\hline Mg II & $16.20 \pm 0.09$ & COG;AOD;FIT & STIS \\
\hline P II & $>15.01$ & COG;FIT & FUSE \\
\hline P III & $13.10 \pm 0.40$ & $\mathrm{AOD}$ & STIS \\
\hline S I & $13.44 \pm 0.07$ & COG;AOD;FIT & STIS \\
\hline $\mathrm{Cl} \mathrm{I}$ & $14.2-14.5^{a}$ & AOD;FIT & STIS+FUSE \\
\hline $\mathrm{Cl}$ II & $14.24 \pm 0.10$ & EW;AOD & FUSE \\
\hline K I & $11.88 \pm 0.04$ & FIT & Optical \\
\hline Ar I & $>14.80$ & FIT & FUSE \\
\hline Mn II & $13.76 \pm 0.07$ & COG;AOD;FIT & STIS \\
\hline Fe II & $15.20 \pm 0.20$ & COG;FIT & FUSE \\
\hline $\mathrm{Ni}$ II & $13.84 \pm 0.15$ & AOD;FIT & STIS \\
\hline $\mathrm{Cu} \mathrm{II}$ & $12.61 \pm 0.09$ & AOD;FIT & STIS \\
\hline Ge II & $13.10 \pm 0.20$ & FIT & STIS \\
\hline $\mathrm{H}_{2}(\mathrm{~J}=0)$ & $20.40 \pm 0.10$ & FIT & FUSE \\
\hline $\mathrm{H}_{2}(\mathrm{~J}=1)$ & $20.50 \pm 0.10$ & FIT & FUSE \\
\hline $\mathrm{H}_{2}(\mathrm{~J}=2)$ & $18.53 \pm 0.10$ & FIT & FUSE \\
\hline $\mathrm{H}_{2}(\mathrm{~J}=3)$ & $17.10 \pm 0.15$ & FIT & FUSE \\
\hline $\mathrm{H}_{2}(\mathrm{~J}=4)$ & $15.40 \pm 0.15$ & FIT & FUSE \\
\hline $\mathrm{H}_{2}(\mathrm{~J}=5)$ & $15.02 \pm 0.20$ & FIT & FUSE \\
\hline $\mathrm{CO}$ & $14.20 \pm 0.20$ & COG;AOD;FIT & FUSE+STIS \\
\hline H I & $21.32 \pm 0.12^{b}$ & & IUE \\
\hline
\end{tabular}

\footnotetext{
${ }^{\text {a }}$ Indicates the methods used to derive the column densities (N): the curve of growth (COG), Apparent Optical Depth (AOD), Equivalent Width (EW) and the profile fitting code "Owens" (FIT). When multiple methods were used, each column density was weighted by its error to produce the weighted means reported here. The associated errors are $1 \sigma$. See chlorine discussion in $\S 4.6$.
}

b From Diplas \& Savage (1994) 
Table 4. Depletions in the LVCs toward HD 192639.

\begin{tabular}{lccr}
\hline \hline Species & $\log (\mathrm{X} / \mathrm{H})_{\odot}^{\mathrm{a}}$ & $\log (\mathrm{X} / \mathrm{H})$ & Depletion $^{b}(1 \sigma)$ \\
\hline $\mathrm{C}$ & -3.45 & - & $-0.40(0.10)$ \\
$\mathrm{O}$ & -3.26 & -3.46 & $-0.20(0.04)$ \\
$\mathrm{Na}$ & -6.29 & - & $-0.60(0.10)$ \\
$\mathrm{Mg}$ & -4.42 & -5.31 & $-0.89(0.06)$ \\
$\mathrm{S}$ & -4.73 & - & $0.00(0.10)$ \\
$\mathrm{Cl}$ & -6.73 & $-6.94^{c}$ & $-0.21\left({ }_{-0.06}^{+0.11}\right)$ \\
$\mathrm{K}$ & -7.57 & - & $-0.70(0.10)$ \\
$\mathrm{Mn}$ & -6.47 & -7.75 & $-1.28(0.07)$ \\
$\mathrm{Fe}$ & -4.49 & -6.31 & $-1.82(0.08)$ \\
$\mathrm{Ni}$ & -5.75 & -7.67 & $-1.92(0.08)$ \\
$\mathrm{Cu}$ & -7.73 & -8.90 & $-1.17(0.06)$ \\
$\mathrm{Ge}$ & -8.37 & -8.41 & $-0.04(0.20)$ \\
\hline
\end{tabular}

a Solar meteoritic and photospheric (C) abundances from Anders \& Grevesse (1989) and Grevesse \& Noels (1993); O abundance from Holweger (2001); logarithmic with $\mathrm{H}=12.00$.

b $1 \sigma$ errors are mentioned in parenthesis. The $(-)$ marks the species for which the depletion was assumed at the value in column (4). For those species a typical error of 0.10 was also assumed.

${ }^{\mathrm{c}}$ Estimate of the abundance and depletion of chlorine using $\log N(\mathrm{Cl} \mathrm{I})=$ 14.3 dex (see $\S 4.6)$. 
Table 5. Electron density in the LVCs toward HD 192639.

\begin{tabular}{lcccccc}
\hline \hline Species & $\begin{array}{c}\log \mathrm{N}(\mathrm{X} \mathrm{I}) \\
(\mathrm{dex})\end{array}$ & $\begin{array}{c}\log \mathrm{N}(\mathrm{X} \mathrm{II})^{b} \\
(\mathrm{dex})\end{array}$ & $\begin{array}{c}\Gamma / \alpha^{a}(\mathrm{WJ} 1) \\
\left(\mathrm{cm}^{-3}\right)\end{array}$ & $\begin{array}{c}n_{e}(\mathrm{WJ} 1) \\
\left(\mathrm{cm}^{-3}\right)\end{array}$ & $\begin{array}{c}\Gamma / \alpha^{a}(\mathrm{D}) \\
\left(\mathrm{cm}^{-3}\right)\end{array}$ & $\begin{array}{c}n_{e}(\mathrm{D}) \\
\left(\mathrm{cm}^{-3}\right)\end{array}$ \\
\hline $\mathrm{Na}$ & $13.90 \pm 0.05$ & $(15.20 \pm 0.10)$ & 2.2 & 0.112 & 2.6 & 0.132 \\
$\mathrm{Mg}$ & $\geq 14.09$ & $16.20 \pm 0.07$ & 13.5 & 0.106 & 12.1 & 0.094 \\
$\mathrm{~K}$ & $11.85 \pm 0.02$ & $(13.95 \pm 0.05)$ & 10.1 & 0.087 & 11.4 & 0.099 \\
$\mathrm{C}$ & $15.29 \pm 0.07$ & $(17.66 \pm 0.05)$ & 24.0 & 0.102 & 40.0 & 0.171 \\
$\mathrm{~S}$ & $13.44 \pm 0.07$ & $(16.78 \pm 0.03)$ & 74.0 & 0.034 & 114.0 & 0.052 \\
\hline
\end{tabular}

${ }^{a} \Gamma / \alpha$ from Aldrovandi \& Péquignot (1973), Aldrovandi \& Péquignot (1974), Péquignot \& Aldrovandi (1986) and Shull \& van Steenberg (1982). $\alpha$ is calculated for $\mathrm{T}=100 \mathrm{~K}$. (WJ1) and (D) refers to the average radiation field as in Witt \& Johnson (1973) and Draine (1978), respectively.

b The parenthesis mark the species for which the depletion was assumed (see Table 4). Errors are $1 \sigma$. 
Table 6. Column Densities of the HVCs TOWARD HD 192639.

\begin{tabular}{|c|c|c|c|}
\hline Species & $\begin{array}{c}\lambda \\
(\AA)\end{array}$ & $\begin{array}{l}\log N \\
(\operatorname{dex})^{*}\end{array}$ & Dataset \\
\hline C III & 977.020 & $>13.50$ & FUSE \\
\hline C IV & 1548.188 & $13.79_{-0.15}^{+0.08}$ & $\mathrm{IUE}^{a}$ \\
\hline N I & 1200.230 & $12.77_{-0.48}^{+0.29}$ & STIS \\
\hline N II & 1084.584 & {$[15.79]$} & FUSE+STIS \\
\hline N II* & 1084.584 & $14.40_{-0.20}^{+0.14}$ & FUSE \\
\hline $\mathrm{Al}$ III & 1862.790 & $13.02_{-0.15}^{+0.14}$ & $\mathrm{IUE}^{a}$ \\
\hline Si II & 1020.667 & $14.62_{-0.17}^{+0.12}$ & FUSE \\
\hline Si II* & 1264.738 & $12.31_{-0.04}^{+0.03}$ & STIS \\
\hline Si IV & 1402.770 & $13.39_{-0.15}^{+0.14}$ & $\mathrm{IUE}^{a}$ \\
\hline S II & 1250.535 & $14.54_{-0.04}^{+0.04}$ & STIS \\
\hline S II & 1253.811 & $14.28_{-0.05}^{+0.04}$ & STIS \\
\hline S II & 1259.519 & $14.50_{-0.06}^{+0.02}$ & STIS \\
\hline Fe II & 2344.213 & $12.95_{-0.26}^{+0.17}$ & $\mathrm{IUE}^{a}$ \\
\hline Fe II & 2374.458 & $12.95_{-0.26}^{+0.17}$ & $\mathrm{IUE}^{a}$ \\
\hline
\end{tabular}

* All FUSE and STIS column densities were derived using the AOD method in the velocity range $[-100,-50] \mathrm{km} \mathrm{s}^{-1}$. Errors are $1 \sigma$. Values in brackets are estimates (see $\S 5.3$ ).

${ }^{\text {a }}$ From Phillips et al. (1984) 